\title{
DERIVATION AND ANALYSIS OF COUPLED PDES ON MANIFOLDS WITH HIGH DIMENSIONALITY GAP ARISING FROM TOPOLOGICAL MODEL REDUCTION*
}

\author{
FEDERICA LAURINo ${ }^{1,2}$ AND PAOlO Zunino ${ }^{1}$
}

\begin{abstract}
Multiscale methods based on coupled partial differential equations defined on bulk and embedded manifolds are still poorly explored from the theoretical standpoint, although they are successfully used in applications, such as microcirculation and flow in perforated subsurface reservoirs. This work aims at shedding light on some theoretical aspects of a multiscale method consisting of coupled partial differential equations defined on one-dimensional domains embedded into three-dimensional ones. Mathematical issues arise because the dimensionality gap between the bulk and the inclusions is larger than one, that is the high dimensionality gap case. First, we show that such model derives from a system of fully three-dimensional equations, by the application of a topological model reduction approach. Secondly, we rigorously analyze the problem, showing that the averaging operators applied for the model reduction introduce a regularization effect that resolves the issues due to the singularity of solutions and to the ill-posedness of restriction operators. Then, we exploit the structure of the model reduction technique to analyze the modeling error. This study confirms that for infinitesimally small inclusions, the modeling error vanishes. Finally, we discretize the problem by means of the finite element method and we analyze the approximation and the model error by means of numerical experiments.
\end{abstract}

1991 Mathematics Subject Classification. 35J25, 58J05, 58C05, 65N30, 65N15, 65N85.

The dates will be set by the publisher.

\section{INTRODUCTION}

The topological (or geometrical) model reduction techniques play an essential role in the simulation of multiscale, multiphysics, multimodel systems. For example, small inclusions of a continuum can be described as zero-dimensional (0D) or one-dimensional (1D) concentrated sources in order to reduce the computational cost of simulations. Many problems in this area are not well investigated yet, such as the coupling of threedimensional (3D) continua with embedded (1D) networks, although it arises in applications of paramount importance. For example, such models have been introduced since three decades (at least), for modeling wells in subsurface reservoirs in $[35,36]$ and for modeling microcirculation in $[5,17,18,42]$. A similar approach has been recently used to model soil/root interactions [23]. However, these application-driven seminal ideas were not followed by a systematic theory and rigorous mathematical analysis.

Keywords and phrases: embedded geometric multiscale, heterogeneous domain dimensionality, model error analysis;

* The authors are members of the INdAM Research group GNCS. The author Federica Laurino acknowledges the support of the Italian Institute of Technology with the fellowship: Sviluppo di metodi computazionali di tipo multi-scala per la simulazione del trasporto vascolare ed extra vascolare di molecole, nano-costrutti e cellule in tessuti neoplastici.

${ }^{1}$ MOX, Department of Mathematics, Politecnico di Milano;

e-mail: federica.laurino@polimi.it \& paolo.zunino@polimi.it

2 Italian Institute of Technology, Genova, Italy 
Seminal works featuring the formulation and analysis of bulk 3D with embedded 1D PDEs, due to D'Angelo and co-workers [12-14,30], have appeared much later than their applications. From the point of view of numerical approximation and analysis of such problems, besides the works by D'Angelo, we mention $[4,6,24-28,33]$. More precisely, in $[4,24,25]$ optimal a-priori error estimates for the finite element approximation of elliptic equations in with Dirac sources are addressed. The solution of 1D differential equations embedded in 2D is studied in [27], recently extended to 3D-1D in [28]. The consistent derivation of numerical approximations schemes for PDEs in mixed dimension is addressed in [6], while [33] focuses on the approximation of 3D-1D coupled problems with mixed finite elements. Relevant topics related to the $3 \mathrm{D}-1 \mathrm{D}$ coupling have also been addressed in $[21,44]$, where regularization of singularities is studied for PDEs with singular source terms.

We believe that these studies do not cover the many facets of a comprehensive theory of coupled PDEs on embedded manifolds with heterogeneous dimensionality. The main issues consist in the poor regularity of solutions of (second order, elliptic) PDEs with singular forcing terms, combined with the ill-posedness of restriction operators (such as the trace operator) applied on manifolds with co-dimension larger than one. We envision different ways to overcome this theoretical obstacle. One is the application of weighted Sobolev spaces to handle singular functions, already adopted in $[13,14]$. The other one is the introduction of regularizing operators. This work explores the latter path, with the main purpose to extend the work of [26] to the more challenging case of 3D-1D coupled equations.

We start from a system of fully 3D second order elliptic equations, coupled by means of Robin type (or mixed type) interface conditions that mimic the flux type interface conditions that are typically used in the applications cited above. This represents an idealized problem of transport/flow through a network of small inclusions embedded into a bulk domain. We identify three general assumptions that allow us to transform the original problem into a simpler one, by means of a topological model reduction technique based on local averaging. The resulting equations consist of 3D-1D coupled elliptic equations. Since the mathematical tools used for the derivation of the reduced model are related to the geometric multiscale method, see for example [39] for a recent review, but are applied here to model small inclusions embedded into a bulk, we call this new approach the geometric embedded multiscale method.

We show that the averaging operators introduce in the problem a regularizing effect, such that the weak solution exists in classical Hilbert spaces. As a consequence, the reduced problem can be naturally approximated by means of finite elements and, thanks to some additional regularity results, the convergence of the approximation method can be proved and the corresponding rates are observed in numerical experiments. We notice that if the small inclusions are shaped as a network, the 1D subproblem consists of a quantum graph, see [3] for details, and its finite element approximation can be addressed with the tools recently developed in [2]. Furthermore, the systematic derivation of the reduced model reveals the structure of the modeling error, allowing us to perform a rigorous analysis of it. This analysis ends up with the conclusion that for infinitesimally small inclusions, the reduced model is equivalent to the fully $3 \mathrm{D}$ one.

We believe that building sound mathematical foundations for the 3D-1D approach to model embedded inclusions will benefit to the theoretical knowledge and it will also improve the reliability of the method for relevant applications, as for example the ones already addressed by the authors and co-workers in $[9,10,31$, $32,37,38]$, see Figure 1 (right panel) for an illustration of these results.

\section{Problem Setting}

We address here the geometrical configuration of the domain and we set up a problem based on RobinNeumann coupling conditions. Figure 1 (left panel) shows a qualitative sketch of the problem setting. Then, we present the formal derivation of the reduced problem, consisting of 3D-1D coupled equations. A rigorous analysis of it will be considered in Section 3.

\subsection{Geometrical setting}

The domain is denoted as $\Omega$ and composed by two parts, $\Sigma$ and $\Omega_{\oplus}:=\Omega \backslash \bar{\Sigma}$. We assume $\Omega$ is convex and $\Sigma$ is completely embedded into $\Omega$, such that the distance between $\partial \Omega$ and $\partial \Sigma$ is strictly positive.

Let $\Sigma$ be a generalized cylinder, that is the swept volume of a two dimensional set moved along a curve in the three-dimensional space, see for example [19]. More precisely, let $\boldsymbol{\lambda}(s)=[\xi(s), \tau(s), \zeta(s)], s \in(0, S)$ be a 

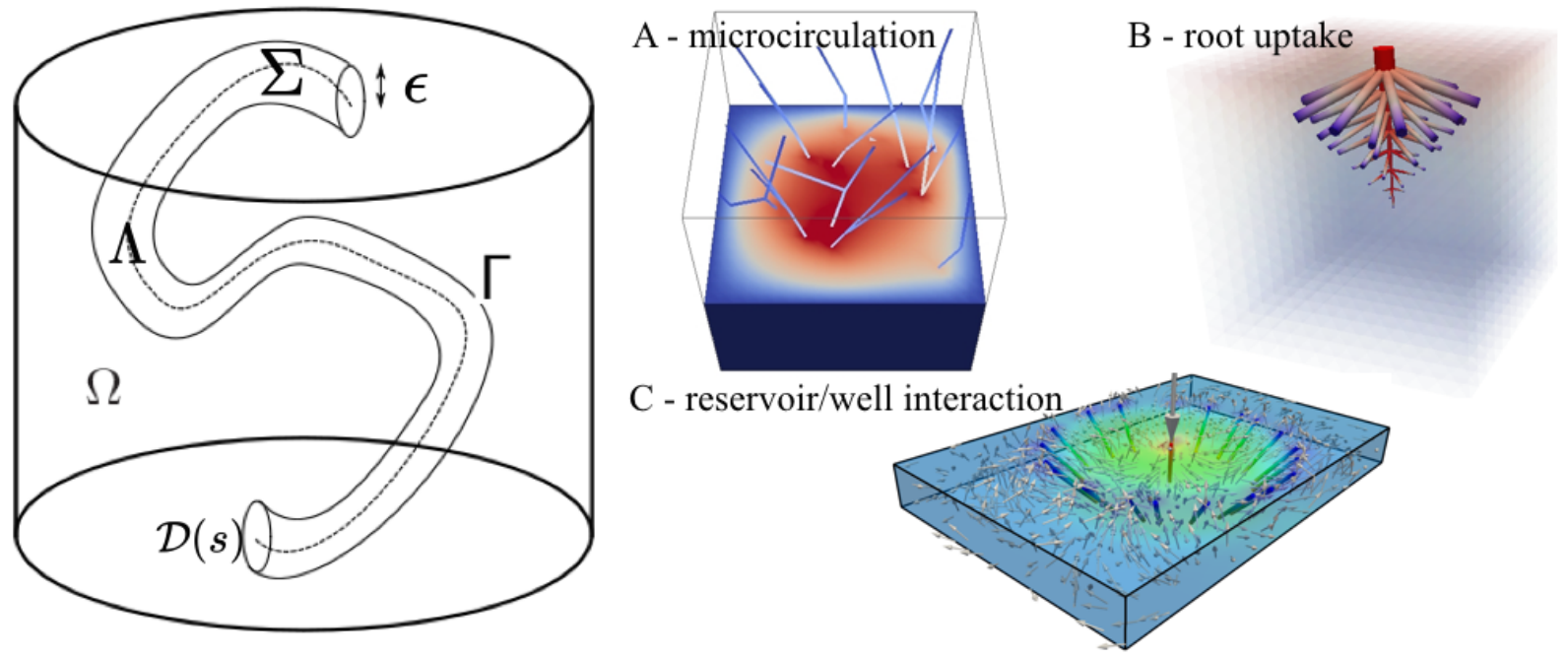

FiguRE 1. The left panel shows a qualitative sketch of the geometrical setting of the problem. The right panel illustrates three significant applications for which the geometric embedded multiscale method has been already successfully used: case (A) represents microcirculation, addressed in $[9,10,31,32,37,38]$; case (B) shows a preliminary study of the interaction of soil and roots pursued in [40]; case $(\mathrm{C})$ represents a prototype simulation for the interaction of reservoirs and wells performed with the approach described in [11].

$\mathcal{C}^{2}$-regular curve in the three-dimensional space. Let $\Lambda=\{\boldsymbol{\lambda}(s), s \in(0, S)\}$ be the centerline of the cylinder. For simplicity, let us assume that $\left\|\boldsymbol{\lambda}^{\prime}(s)\right\|=1$ such that the arc-length and the coordinate $s$ coincide. Let $\boldsymbol{T}, \boldsymbol{N}, \boldsymbol{B}$ be the Frenet frame related to the curve.

Let $\mathcal{D}(s)=[x(r, t), y(r, t)]:(0, R(s)) \times(0, T(s)) \rightarrow \mathbb{R}^{2}$ be a parametrization of the cross section. Let us assume that $\mathcal{D}(s)$ is convex for any $s \in(0, S)$. The cross section can change size along $\Lambda$ but not shape. Let us also parametrize the boundary of the cross section as $\partial \mathcal{D}(s)=[\partial x(r, t), \partial y(r, t)]:(0, R(s)) \times(0, T(s)) \rightarrow \mathbb{R}^{2}$, and let us assume that $\partial \mathcal{D}(s)$ is a piecewise $\mathcal{C}^{2}$-regular curve. Then, the generalized cylinder $\Sigma$ can be defined as follows

$$
\Sigma=\{\boldsymbol{\lambda}(s)+x(r, t) \boldsymbol{N}(s)+y(r, t) \boldsymbol{B}(s), r \in(0, R(s)), s \in(0, S), t \in(0, T(s))\},
$$

and the lateral boundary of it, denoted with $\Gamma$, is

$$
\Gamma=\{\boldsymbol{\lambda}(s)+\partial x(r, t) \boldsymbol{N}(s)+\partial y(r, t) \boldsymbol{B}(s), r \in(0, R(s)), s \in(0, S), t \in(0, T(s))\} .
$$

Let $|\cdot|$ denote the Lebesgue measure of a set. If $|\mathcal{D}(0)|,|\mathcal{D}(S)|>0$ then $\Sigma$ has top and bottom boundaries, which are $\partial \Sigma \backslash \Gamma=\{\boldsymbol{\lambda}(0)+\mathcal{D}(0)\} \cup\{\boldsymbol{\lambda}(S)+\mathcal{D}(S)\}$. For simplicity of notation we write $\Gamma_{0}=\{\boldsymbol{\lambda}(0)+\mathcal{D}(0)\}$ and $\Gamma_{S}=\{\boldsymbol{\lambda}(S)+\mathcal{D}(S)\}$.

Thanks to the geometrical structure of the generalized cylinder, we decompose integrals as follows, for any sufficiently regular function $w$,

$$
\begin{gathered}
\int_{\Sigma} w d \omega=\int_{\Lambda} \int_{\mathcal{D}(s)} w d \sigma d s=\int_{\Lambda}|\mathcal{D}(s)| \overline{\bar{w}}(s) d s, \text { where } \overline{\bar{w}}(s)=|\mathcal{D}(s)|^{-1} \int_{\mathcal{D}(s)} w d \sigma \\
\int_{\partial \Sigma} w d \sigma=\int_{\Lambda} \int_{\partial \mathcal{D}(s)} w d \gamma d s=\int_{\Lambda}|\partial \mathcal{D}(s)| \bar{w}(s) d s, \text { where } \bar{w}(s)=|\partial \mathcal{D}(s)|^{-1} \int_{\partial \mathcal{D}(s)} w d \gamma
\end{gathered}
$$

being $d \omega, d \sigma, d \gamma$ the generic volume, surface and curvilinear Lebesgue measures.

With little abuse of notation, for a straight cylinder we identify the function $\overline{\bar{w}}(s): \Lambda \rightarrow \mathbb{R}$ with the function on $\Sigma$ obtained by extending the mean value to each cross section $\mathcal{D}(s)$. The same extension can be also applied to $\Gamma$, namely $\bar{w}(s)$ can be either regarded as a function on $\Lambda$ or on $\Gamma$.

Let us now formulate a fundamental assumption (A0) on the proportions of $\Sigma$. 
A0: We assume that the transversal diameter of $\Sigma$ is small compared to the diameter of $\Omega$. The small parameter (defined below) is denoted with the symbol $\epsilon$.

Let $D=\max _{s \in(0, S)} \operatorname{diam}(\mathcal{D}(s))$ be the largest diameter of the cross sections of $\Sigma$, that is the transversal diameter of $\Sigma$. The central assumption of this work is that $D \ll \operatorname{diam}(\Omega)$. Let us now scale the domains $\Omega$ and $\Sigma$. Let $\chi_{\Omega}(\boldsymbol{x})=\boldsymbol{x} / \operatorname{diam}(\Omega)$ be a scaling function and let be $\Omega_{\chi}=\chi_{\Omega}(\Omega), \Sigma_{\chi}=\chi_{\Omega}(\Sigma)$ be the scaled domains. The previous assumption implies that for the scaled domains $\epsilon=D_{\chi}=D / \operatorname{diam}(\Omega)$ is such that $0<\epsilon \ll 1$. For simplicity of notation, and without loss of generality, form now on we will implicitly refer to the scaled domains dropping the subindex $\chi$.

\subsection{Fully 3D coupled problem formulation}

We describe here a version of the problem arising from Robin-Neumann conditions. After straightforwardly rearranging the Robin-Neumann conditions as Robin-Robin ones, the problem consists to find $u_{\oplus}, u_{\ominus}$ (where $\oplus, \ominus$ denote the exterior and the interior of $\Sigma$, respectively) such as:

$$
\begin{array}{ll}
-\Delta u_{\oplus}=f & \text { in } \Omega_{\oplus}, \\
-\Delta u_{\ominus}=g & \text { in } \Sigma, \\
-\nabla u_{\oplus} \cdot \boldsymbol{n}_{\oplus}=\kappa\left(u_{\oplus}-u_{\ominus}\right) & \text { on } \Gamma, \\
-\nabla u_{\ominus} \cdot \boldsymbol{n}_{\ominus}=\kappa\left(u_{\ominus}-u_{\oplus}\right) & \text { on } \Gamma, \\
-\nabla u_{\oplus} \cdot \boldsymbol{n}_{\oplus}=0 & \text { on } \Gamma_{0} \cup \Gamma_{S}, \\
-\nabla u_{\ominus} \cdot \boldsymbol{n}_{\ominus}=0 & \text { on } \Gamma_{0} \cup \Gamma_{S}, \\
\quad u_{\oplus}=0 & \text { on } \partial \Omega .
\end{array}
$$

It is assumed that the interface of $\Sigma$ is permeable, namely it is crossed by a normal flux proportional to $\kappa\left(u_{\oplus}-u_{\ominus}\right)$. The coefficient $\kappa$ plays the role of permeability or transfer coefficient and it assumes a uniform value on each cross section $\partial \mathcal{D}(s)$. As a result of that, $\kappa$ is only a (regular) function of the arc-length $s$. For the boundary conditions on the top and bottom faces of the cylinder, we make the assumption that $|\mathcal{D}(0)|,|\mathcal{D}(S)|>0$. In applications, the domain $\Sigma$ consists of many cylinders, representing channels carrying flow, fibers or inclusions. The numerical approximation of PDEs on such domain may thus become very demanding, due to its complex shape. The main disadvantage is that it requires the resolution of the full geometry of the inclusions, which in many real applications can be difficult to handle. For this reason, we aim to apply topological model reduction techniques, based on averaging, in order to transform the problem on $\Sigma$ into a simpler one.

The objective of this work is to consider a simplified version of problem (1), where the domain $\Sigma$ shrinks to its centerline $\Lambda$ and the corresponding partial differential equation is averaged on the cylinder cross section, namely $\mathcal{D}$. This new problem setting will be called the reduced problem. From the mathematical standpoint it is more challenging than (1), because it involves the coupling of $3 \mathrm{D} / 1 \mathrm{D}$ elliptic problems.

\subsection{Topological model reduction of the problem on $\Sigma$}

We apply the averaging technique to equation (1b). In particular, we consider an arbitrary portion $\mathcal{P}$ of the cylinder, with lateral surface $\Gamma_{\mathcal{P}}$ and bounded by two perpendicular sections to $\Lambda$, namely $\mathcal{D}\left(s_{1}\right), \mathcal{D}\left(s_{2}\right)$ with $s_{1}<s_{2}$. We have,

$$
\int_{\mathcal{P}} \Delta u_{\ominus} d \omega=\int_{\partial \mathcal{P}} \nabla u_{\ominus} \cdot \boldsymbol{n}_{\ominus} d \sigma=-\int_{\mathcal{D}\left(s_{1}\right)} \partial_{s} u_{\ominus} d \sigma+\int_{\mathcal{D}\left(s_{2}\right)} \partial_{s} u_{\ominus} d \sigma+\int_{\Gamma_{\mathcal{P}}} \nabla u_{\ominus} \cdot \boldsymbol{n}_{\ominus} d \sigma .
$$

By the fundamental theorem of integral calculus combined with the Reynolds transport Theorem, we have

$$
\begin{aligned}
-\int_{\mathcal{D}\left(s_{1}\right)} \partial_{s} u_{\ominus} d \sigma+\int_{\mathcal{D}\left(s_{2}\right)} \partial_{s} u_{\ominus} d \sigma & =\int_{s_{1}}^{s_{2}} d_{s} \int_{\mathcal{D}(s)} \partial_{s} u_{\ominus} d \sigma d s \\
& =\int_{s_{1}}^{s_{2}} d_{s s}^{2} \int_{\mathcal{D}(s)} u_{\ominus} d \sigma d s-\int_{s_{1}}^{s_{2}} d_{s}\left(\int_{\partial \mathcal{D}(s)} \nu u_{\ominus} d \gamma\right) d s
\end{aligned}
$$


where $\nu$ denotes the normal deformation of the boundary along $(0, S)$. More precisely, we observe that $\nu$ is uniform on $\partial \mathcal{D}(s)$, because we assume that $\mathcal{D}(s)$ can not change shape. Then, the following identity holds true,

$$
d_{s}(|\mathcal{D}(s)|)=\int_{\partial \mathcal{D}(s)} \nu d \gamma=|\partial \mathcal{D}(s)| \nu
$$

Substituting in the previous equality, we obtain

$$
\begin{aligned}
\int_{s_{1}}^{s_{2}} d_{s s}^{2} \int_{\mathcal{D}(s)} u_{\ominus} d \sigma d s-\int_{s_{1}}^{s_{2}} d_{s}\left(\int_{\partial \mathcal{D}(s)} \nu u_{\ominus} d \gamma\right) d s & =\int_{s_{1}}^{s_{2}}\left[d_{s s}^{2}\left(|\mathcal{D}(s)| \overline{\bar{u}}_{\ominus}\right)-d_{s}\left(\nu|\partial \mathcal{D}(s)| \bar{u}_{\ominus}\right)\right] d s \\
& =\int_{s_{1}}^{s_{2}}\left[d_{s s}^{2}\left(|\mathcal{D}(s)| \overline{\bar{u}}_{\ominus}\right)-d_{s}\left(d_{s}(|\mathcal{D}(s)|) \bar{u}_{\ominus}\right)\right] d s
\end{aligned}
$$

By means of (1d) we obtain,

$$
\int_{\Gamma_{\mathcal{P}}} \nabla u_{\ominus} \cdot \boldsymbol{n}_{\ominus} d \sigma=-\int_{\Gamma_{\mathcal{P}}} \kappa\left(u_{\ominus}-u_{\oplus}\right) d \sigma=-\int_{s_{1}}^{s_{2}} \int_{\partial \mathcal{D}(s)} \kappa\left(u_{\ominus}-u_{\oplus}\right) d \gamma d s=-\int_{s_{1}}^{s_{2}} \kappa|\partial \mathcal{D}(s)|\left(\bar{u}_{\ominus}-\bar{u}_{\oplus}\right) d s .
$$

From the combination of all the above terms with the right hand side, we obtain that the solution $u_{\ominus}$ of (1) satisfies,

$$
\int_{s_{1}}^{s_{2}}\left[-d_{s s}^{2}\left(|\mathcal{D}(s)| \overline{\bar{u}}_{\ominus}\right)+d_{s}\left(d_{s}(|\mathcal{D}(s)|) \bar{u}_{\ominus}\right)+|\partial \mathcal{D}(s)| \kappa\left(\bar{u}_{\ominus}-\bar{u}_{\oplus}\right)\right] d s=\int_{s_{1}}^{s_{2}}|\mathcal{D}(s)| \overline{\bar{g}} d s .
$$

Since the choice of the points $s_{1}, s_{2}$ is arbitrary, we conclude that the following equation holds true,

$$
-d_{s s}^{2}\left(|\mathcal{D}(s)| \overline{\bar{u}}_{\ominus}\right)+d_{s}\left(d_{s}(|\mathcal{D}(s)|) \bar{u}_{\ominus}\right)+|\partial \mathcal{D}(s)| \kappa\left(\bar{u}_{\ominus}-\bar{u}_{\oplus}\right)=|\mathcal{D}(s)| \overline{\bar{g}} \quad \text { on } \Lambda,
$$

which is complemented by the following conditions at the boundary of $\Lambda$,

$$
|\mathcal{D}(s)| d_{s} \overline{\bar{u}}_{\ominus}=0, \quad d_{s}|\mathcal{D}(s)|=0, \quad \text { on } \quad s=0, S .
$$

Then, we consider the variational formulation of the averaged equation (3). After multiplication by a test function $V \in H^{1}(\Lambda)$, integration on $\Lambda$ and suitable application of integration by parts, we obtain,

$$
\begin{aligned}
\int_{\Lambda} d_{s}\left(|\mathcal{D}(s)| \overline{\bar{u}}_{\ominus}\right) d_{s} V d s-\left.d_{s}\left(|\mathcal{D}(s)| \overline{\bar{u}}_{\ominus}\right) V\right|_{s=0} ^{s=S}-\int_{\Lambda}\left(d_{s}|\mathcal{D}(s)|\right) \bar{u}_{\ominus} d_{s} V d s+\left.\left(d_{s}|\mathcal{D}(s)|\right) \bar{u}_{\ominus} V\right|_{s=0} ^{s=S} \\
+\int_{\Lambda}|\partial \mathcal{D}(s)| \kappa\left(\bar{u}_{\ominus}-\bar{u}_{\oplus}\right) V d s=\int_{\Lambda}|\mathcal{D}(s)| \overline{\bar{g}} V d s .
\end{aligned}
$$

Using boundary conditions, the identity $d_{s}\left(|\mathcal{D}(s)| \overline{\bar{u}}_{\ominus}\right)=|\mathcal{D}(s)| d_{s} \overline{\bar{u}}_{\ominus}+d_{s}(|\mathcal{D}(s)|) \overline{\bar{u}}_{\ominus}$ and reminding that $\left.d_{s}|\mathcal{D}(s)|\right) /|\partial \mathcal{D}(s)|=\nu$, we obtain,

$$
\left(d_{s} \overline{\bar{u}}_{\ominus}, d_{s} V\right)_{\Lambda,|\mathcal{D}|}+\left(\nu\left(\overline{\bar{u}}_{\ominus}-\bar{u}_{\ominus}\right), d_{s} V\right)_{\Lambda,|\partial \mathcal{D}|}+\left(\kappa\left(\bar{u}_{\ominus}-\bar{u}_{\oplus}\right), V\right)_{\Lambda,|\partial \mathcal{D}|}=(\overline{\bar{g}}, V)_{\Lambda,|\mathcal{D}|} .
$$

where we have introduced the following weighted inner product notation,

$$
(U, V)_{\Lambda, w}=\int_{0}^{S} w(s) U(s) V(s) d s .
$$

Let us now formulate the modelling assumption that allows us to reduce equation (5) to a solvable onedimensional (1D) model. More precisely, we assume that:

A1: the function $u_{\ominus}$ has a uniform profile on each cross section $\mathcal{D}(s)$, namely $u_{\ominus}(r, s, t)=U(s)$. 
Therefore, observing that $U=\bar{U}=\overline{\bar{U}}$, problem (5) consists to find $U \in H^{1}(\Lambda)$ such that

$$
\left(d_{s} U, d_{s} V\right)_{\Lambda,|\mathcal{D}|}+(\kappa U, V)_{\Lambda,|\partial \mathcal{D}|}=\left(\kappa \bar{u}_{\oplus}, V\right)_{\Lambda,|\partial \mathcal{D}|}+(\overline{\bar{g}}, V)_{\Lambda,|\mathcal{D}|} \quad \forall V \in H^{1}(\Lambda) .
$$

Remark 2.1. By formally writing the strong formulation of equation (6) we obtain,

$$
-d_{s s}^{2} U+\frac{|\partial \mathcal{D}|}{|\mathcal{D}|} \kappa U=\frac{|\partial \mathcal{D}|}{|\mathcal{D}|} \kappa \bar{u}_{\oplus}+\overline{\bar{g}}, \quad \text { on } \Lambda .
$$

We observe that the relative magnitude of the flux terms, that are $(|\partial \mathcal{D}| /|\mathcal{D}|) \kappa\left(U-\bar{u}_{\oplus}\right)$, scales as $(|\partial \mathcal{D}| /|\mathcal{D}|) \kappa$ with respect to the volumetric terms, namely $d_{s s}^{2} U+\overline{\bar{g}}$. This sheds light on the behavior of the model when $\epsilon \rightarrow 0$. More precisely, for a bounded $\kappa$, what matters is the limit of $|\partial \mathcal{D}| /|\mathcal{D}|$. Assuming that $\partial \mathcal{D}$ is rectifiable and the cross section $\mathcal{D}$ is characterized by two axes, of length $\epsilon$ and $\epsilon^{\alpha}$ respectively (where the parameter $\alpha$ controls the aspect ratio of the cross section), we have $\lim _{\epsilon \rightarrow 0}|\partial \mathcal{D}| /|\mathcal{D}| \simeq \epsilon^{-\alpha}+\epsilon^{-1} \simeq \epsilon^{-\max (\alpha, 1)}$. Unless $\alpha>1$, the scaling of $|\partial \mathcal{D}| /|\mathcal{D}|$ is $\epsilon^{-1}$. Going back to the strong formulation of (6), this entails that the flux terms diverge when $\epsilon \rightarrow 0$. This observation explains, from an heuristic yet physical standpoint, why the coupling between the 3D and the 1D models becomes ill posed when the radius of the $1 \mathrm{D}$ inclusion vanishes.

\subsection{Topological model reduction of the problem on $\Omega_{\oplus}$}

We focus here on the subproblem of (1) related to $\Omega_{\oplus}$, that is

$$
\begin{array}{cl}
-\Delta u_{\oplus}=f & \text { in } \Omega_{\oplus}, \\
-\nabla u_{\oplus} \cdot \boldsymbol{n}_{\oplus}=\kappa\left(u_{\oplus}-u_{\ominus}\right) & \text { on } \Gamma, \\
u_{\oplus}=0 & \text { on } \partial \Omega .
\end{array}
$$

First, we multiply both sides of (7a) for a test function $v \in H_{0}^{1}(\Omega)$,

$$
-\int_{\Omega_{\oplus}} \Delta u_{\oplus} v d \omega=\int_{\Omega_{\oplus}} f v d \omega .
$$

Integrating by parts and using boundary and interface conditions, we obtain:

$$
\begin{aligned}
\int_{\Omega_{\oplus}} f v d \omega & =-\int_{\Omega_{\oplus}} \Delta u_{\oplus} v d \omega=\int_{\Omega_{\oplus}} \nabla u_{\oplus} \cdot \nabla v d \omega-\int_{\partial \Omega_{\oplus}} \nabla u_{\oplus} \cdot \boldsymbol{n}_{\oplus} v d \sigma \\
& =\int_{\Omega_{\oplus}} \nabla u_{\oplus} \cdot \nabla v d \Omega+\int_{\Gamma} \kappa\left(u_{\oplus}-u_{\ominus}\right) v d \sigma .
\end{aligned}
$$

Now, we apply a topological model reduction of the interface conditions, namely we go from a 3D-3D to a 3D-1D formulation. To this purpose, let us write the solution and the test functions on every cross section $\partial \mathcal{D}(s)$ as their average plus some fluctuation,

$$
u_{\oplus}=\bar{u}_{\oplus}+\tilde{u}_{\oplus}, \quad u_{\ominus}=\bar{u}_{\ominus}+\tilde{u}_{\ominus}, \quad v=\bar{v}+\tilde{v}, \quad \text { on } \partial \mathcal{D}(s),
$$

where $\overline{\tilde{u}}_{\oplus}=\overline{\tilde{u}}_{\ominus}=\overline{\tilde{v}}=0$. Therefore, using the coordinates system $(r, s, t)$ on $\Gamma$, for $*=\oplus, \ominus$ we have,

$$
\int_{\Gamma} \kappa u_{*} v d \sigma=\int_{\Lambda} \kappa \int_{\partial \mathcal{D}(s)}\left(\bar{u}_{*}+\tilde{u}_{*}\right)(\bar{v}+\tilde{v}) d \gamma d s=\int_{\Lambda} \kappa|\partial \mathcal{D}(s)| \bar{u}_{*} \bar{v} d s+\int_{\Lambda} \kappa \int_{\partial \mathcal{D}(s)} \tilde{u}_{*} \tilde{v} d \gamma d s .
$$

Then, we make the following modelling assumptions:

A2: we identify the domain $\Omega_{\oplus}$ with the entire $\Omega$, and we correspondingly omit the subscript $\oplus$ to the functions defined on $\Omega_{\oplus}$, namely

$$
\int_{\Omega_{\oplus}} u_{\oplus} d \omega \simeq \int_{\Omega} u d \omega .
$$


A3: we assume that the product of fluctuations is small, namely

$$
\int_{\partial \mathcal{D}(s)} \tilde{u}_{*} \tilde{v} d \gamma \simeq 0
$$

By means of the previous deductions, reminding that for assumption A1 we have that $\bar{u}_{\ominus}=U$ and putting together the terms of the weak form of (7), we obtain that $u$ solves the following problem,

$$
(\nabla u, \nabla v)_{\Omega}+(\kappa \bar{u}, \bar{v})_{\Lambda,|\partial \mathcal{D}|}=(\kappa U, \bar{v})_{\Lambda,|\partial \mathcal{D}|}+(f, v)_{\Omega}, \quad \forall v \in H_{0}^{1}(\Omega)
$$

\subsection{Extension of the 1D problem to a metric graph}

The embedded domain $\Sigma$ was defined starting from its centerline, namely the curve $\Lambda$. We now discuss the generalization to the case where $\Lambda$ is a network. In our case, the edges of the network are curves $\boldsymbol{\lambda}_{i}\left(s_{i}\right)=\left[\xi_{i}\left(s_{i}\right), \tau_{i}\left(s_{i}\right), \zeta_{i}\left(s_{i}\right)\right], s_{i} \in\left(0, S_{i}\right), i=1, \ldots, N$ that are connected at a number $\mathrm{M}$ of vertices,

$$
\boldsymbol{y}_{j}=\boldsymbol{\lambda}_{i}(0)=\boldsymbol{\lambda}_{\hat{\imath}}\left(S_{\hat{\imath}}\right), \quad i, \hat{\imath} \in\{1, \ldots, N\}, j=1, \ldots, M
$$

The set of vertices is denoted with $\boldsymbol{Y}=\left\{\boldsymbol{y}_{j} \in \mathbb{R}^{d}, j=1, \ldots, M\right\}$, while $\mathcal{K}_{j}$, represent all the indices $i$ that are connected with the vertex $j$. Furthermore, $\mathcal{K}_{j}$ can be decomposed into $\mathcal{K}_{j}^{-}$and $\mathcal{K}_{j}^{+}$, where $\mathcal{K}_{j}^{-}=\left\{i \in\{1, \ldots, N\}: \boldsymbol{y}_{j}=\boldsymbol{\lambda}_{i}(0)\right\}$ are the branches originating in the $j$-th vertex, according to their orientation. The complementary is $\mathcal{K}_{j}^{+}$denoting the branches that end into the same vertex. We denote with $i \in \mathcal{B}$ the indices of segments with a dead-end, which can be similarly split into $\mathcal{B}^{+}, \mathcal{B}^{-}$. Obviously, each edge has an arc-coordinate $s_{i}$ and a length $S_{i}$, under the assumption $\left\|\boldsymbol{\lambda}_{i}^{\prime}\right\|=1$. With these two properties, the network is also a metric graph. In this more general setting the embedded domain $\Sigma$ is defined as the union of all the generalized cylinders generated by swiping suitable sections $\partial \mathcal{D}\left(s_{i}\right)$ along the centerlines $\Lambda_{i}=\left\{\boldsymbol{\lambda}_{i}\left(s_{i}\right), s_{i} \in\left(0, S_{i}\right)\right\}$, namely $\Sigma=\bigcup_{i=1}^{N} \Sigma_{i}$.

We observe that the topological model reduction approach can still be applied branch by branch individually, but it can not be adapted to the entire $\Sigma$ at once, because in proximity of the junctions $\Sigma$ is no longer a generalized cylinder. For this reason we define the reduced problem directly from the differential formulation of a single branch. Equation (3) applies to each edge $\Lambda_{i}$ and it must be complemented with suitable matching conditions at the vertices, which correspond to the application of (4) at the endpoints of each $\Lambda_{i}$. Such conditions turn out to be the Kirchhoff ones that for the $j$-th vertex can be written as,

$$
\begin{array}{ll}
\sum_{i \in \mathcal{K}_{j}^{+}}\left|\mathcal{D}\left(S_{i}\right)\right| d_{s_{i}} U_{i}\left(S_{i}\right)-\sum_{i \in \mathcal{K}_{j}^{-}}|\mathcal{D}(0)| d_{s_{i}} U_{i}(0)=0, & \\
U_{i}(0)=U_{\hat{\imath}}\left(S_{\hat{\imath}}\right), & \forall i \in \mathcal{K}_{j}^{-}, \hat{\imath} \in \mathcal{K}_{j}^{+}, \\
d_{s_{i}}|\mathcal{D}(0)|=d_{s_{i}}\left|\mathcal{D}\left(S_{i}\right)\right|=0, & \forall i \in \mathcal{K}_{j} .
\end{array}
$$

The first conditions corresponds to balance of current or fluxes, while the second states that the solution on each edge must be continuous at the vertices. The third condition is just an assumption on the shape of each branch at the endpoints. The reduced problem on the network consists to find a collection of functions $U_{i}, i=1, \ldots, N$ such that 


$$
\begin{array}{rlrl}
-d_{s s}^{2}\left(\left|\mathcal{D}\left(s_{i}\right)\right| U_{i}\right)+d_{s_{i}}\left(d_{s_{i}}\left|\mathcal{D}\left(s_{i}\right)\right| U_{i}\right)+\left|\partial \mathcal{D}\left(s_{i}\right)\right| \kappa_{i} U_{i} & & \\
=\left|\partial \mathcal{D}\left(s_{i}\right)\right| \kappa_{i} \bar{u}_{\oplus}+\left|\mathcal{D}\left(s_{i}\right)\right| \overline{\bar{g}}, & & \text { on } \Lambda_{i}, \forall i=1, \ldots, N, \\
d_{s_{i}}|\mathcal{D}(0)|=d_{s_{i}}\left|\mathcal{D}\left(S_{i}\right)\right|=0, & & \forall i \in \mathcal{K}_{j}, \forall j=1, \ldots, M, \\
& \sum_{i \in \mathcal{K}_{j}^{+}}\left|\mathcal{D}\left(S_{i}\right)\right| d_{s_{i}} U_{i}\left(S_{i}\right)-\sum_{i \in \mathcal{K}_{j}^{-}}|\mathcal{D}(0)| d_{s_{i}} U_{i}(0)=0, & & \forall j=1, \ldots, M, \\
U_{i}(0)=U_{\hat{\imath}}\left(S_{\hat{\imath}}\right), & & \forall i \in \mathcal{K}_{j}^{-}, \forall \hat{\imath} \in \mathcal{K}_{j}^{+}, \forall j=1, \ldots, M, \\
|\mathcal{D}(0)| d_{s_{i}} U_{i}(0)=0, d_{s_{i}}|\mathcal{D}(0)|=0, & & \forall i \in \mathcal{B}^{-}, \\
\left|\mathcal{D}\left(S_{i}\right)\right| d_{s_{i}} U_{i}\left(S_{i}\right)=0, d_{s_{i}}\left|\mathcal{D}\left(S_{i}\right)\right|=0, & & \forall i \in \mathcal{B}^{+} .
\end{array}
$$

For the definition of the variational formulation of problem (9) we introduce Sobolev spaces defined on a metric graph, see for example [2,43] and references therein. In particular $H^{1}(\Lambda)=\bigoplus_{\Lambda_{i}} H^{1}\left(\Lambda_{i}\right) \cap \mathcal{C}^{0}(\Lambda)$, namely the space of all continuous functions $V: \Lambda \rightarrow \mathbb{R}$, such that their restriction $V_{i}$ to each edge $\Lambda_{i}, i=1, \ldots, N$ belongs to $H^{1}\left(\Lambda_{i}\right)$, where $H^{1}\left(\Lambda_{i}\right)$ denotes the usual $H^{1}$ space defined on the manifold $\Lambda_{i}$. The norm of $H^{1}(\Lambda)$ is naturally defined as, $\|V\|_{H^{1}(\Lambda)}^{2}=\sum_{i=1}^{N}\left\|V_{i}\right\|_{H^{1}\left(\Lambda_{i}\right)}^{2}$.

Let us now take $U, V \in H^{1}(\Lambda)$ and derive the variational formulation of (9). From (9a), we obtain,

$$
\begin{aligned}
\sum_{i=1}^{N}\left[\left(d_{s_{i}} U_{i}, d_{s} V_{i}\right)_{\Lambda_{i},|\mathcal{D}|}-\left.d_{s_{i}}\left(\left|\mathcal{D}\left(s_{i}\right)\right| U_{i}\right) V_{i}\right|_{s_{i}=0} ^{s_{i}=S_{i}}+\left.d_{s_{i}}\left(\left|\mathcal{D}\left(s_{i}\right)\right|\right) U_{i} V_{i}\right|_{s_{i}=0} ^{s_{i}=S_{i}}+\left(\kappa_{i} U_{i}, V_{i}\right)_{\Lambda_{i},|\partial \mathcal{D}|}\right] & \\
& =\sum_{i=1}^{N}\left(\kappa_{i} \bar{u}_{\oplus}, V_{i}\right)_{\Lambda_{i},|\partial \mathcal{D}|}+(\overline{\bar{g}}, V)_{\Lambda,|\mathcal{D}|}
\end{aligned}
$$

After using (9b) and reordering the terms at the endpoints of each edge we have,

$$
\begin{aligned}
& \sum_{i=1}^{N}\left[\left|\mathcal{D}\left(S_{i}\right)\right| d_{s_{i}} U_{i}\left(S_{i}\right)\right.\left.V_{i}\left(S_{i}\right)-|\mathcal{D}(0)| d_{s_{i}} U_{i}(0) V_{i}(0)\right] \\
&=\sum_{j=1}^{M}\left[\sum_{i \in \mathcal{K}_{j}^{+}}\left|\mathcal{D}\left(S_{i}\right)\right| d_{s_{i}} U_{i}\left(S_{i}\right) V_{i}\left(S_{i}\right)-\sum_{i \in \mathcal{K}_{j}^{-}}|\mathcal{D}(0)| d_{s_{i}} U_{i}(0) V_{i}(0)\right] \\
& \quad+\sum_{i \in \mathcal{B}^{+}}\left|\mathcal{D}\left(S_{i}\right)\right| d_{s_{i}} U_{i}\left(S_{i}\right) V_{i}\left(S_{i}\right)-\sum_{i \in \mathcal{B}^{-}}|\mathcal{D}(0)| d_{s_{i}} U_{i}(0) V_{i}(0) .
\end{aligned}
$$

Since the test functions are continuous on $\Lambda$, they can be factorized and all the terms on $\mathcal{K}_{j}$ and $\mathcal{B}$ disappear owing to (9c)-(9e)-(9f). Finally, conditions (9d) are stongly enforced through the definition of $H^{1}(\Lambda)$. As a result of that, the variational formulation of the reduced problem on the network consists of finding $U \in H^{1}(\Lambda)$ such that

$$
\sum_{i=1}^{N}\left[\left(d_{s_{i}} U_{i}, d_{s} V_{i}\right)_{\Lambda_{i},|\mathcal{D}|}+\left(\kappa_{i} U_{i}, V_{i}\right)_{\Lambda_{i},|\partial \mathcal{D}|}\right]=\sum_{i=1}^{N}\left(\kappa_{i} \bar{u}_{\oplus}, V_{i}\right)_{\Lambda_{i},|\partial \mathcal{D}|}+(\overline{\bar{g}}, V)_{\Lambda,|\mathcal{D}|}
$$

Endowed with problem (10) the metric graph $\Lambda$ becomes a quantum graph, namely a metric graph equipped with a differential operator on the edges complemented with vertex conditions, see for example [3]. In the simple case of constant cross sections, namely $d_{s}|\partial \mathcal{D}(s)|=0$, the differential operator on $\Lambda$ is $\mathcal{L}(U)=$ $|\mathcal{D}| d_{s s}^{2} U+|\partial \mathcal{D}| \kappa U$ (also called as a Schrodinger-type or Hamiltonian operator) and the vertex conditions are the Kirchhoff equations reported above. 


\section{Mathematical ANALYsis AND NUMERICAL APPROXimation of THE PROBLEM}

We study the existence, uniqueness, stability and regularity of solutions of problem (6)-(8). The main focus of this section is to show how the formal derivation of the 3D-1D coupled problem actually provides a mathematically sound definition of the coupling operators from a $3 \mathrm{D}$ domain, $\Omega$, to a $1 \mathrm{D}$ manifold, $\Lambda$, and vice versa. This is a non trivial result, because the standard trace operator form a domain $\Omega$ to a subset $\Lambda$ is not well posed if $\Lambda$ is a manifold of co-dimension two of $\Omega$.

\subsection{Coupled problems with hybrid dimensionality}

Let us now introduce the following bilinear forms:

$$
\begin{aligned}
& a_{\Omega}(w, v)=(\nabla w, \nabla v)_{\Omega}, \\
& a_{\Lambda}(w, v)=\left(d_{s} w, d_{s} v\right)_{\Lambda,|\mathcal{D}|}, \\
& b_{\Lambda}^{\epsilon}(w, v)=(\kappa w, v)_{\Lambda,|\partial \mathcal{D}|} .
\end{aligned}
$$

After averaging of the equation on $\Omega_{\ominus}$ and of the interface conditions, for any $f \in L^{2}(\Omega), \overline{\bar{g}} \in L^{2}(\Lambda)$, the weak formulation of the reduced problem consists to find $u \in H_{0}^{1}(\Omega), U \in H^{1}(\Lambda)$ such that

$$
\begin{array}{ll}
a_{\Omega}(u, v)+b_{\Lambda}^{\epsilon}(\bar{u}, \bar{v})=b_{\Lambda}^{\epsilon}(U, \bar{v})+(f, v)_{\Omega} & \forall v \in H_{0}^{1}(\Omega), \\
a_{\Lambda}(U, V)+b_{\Lambda}^{\epsilon}(U, V)=b_{\Lambda}^{\epsilon}(\bar{u}, V)+(\overline{\bar{g}}, V)_{\Lambda,|\mathcal{D}|} & \forall V \in H^{1}(\Lambda) .
\end{array}
$$

This problem is an extension to 3D of the one considered in [26] for two space dimensions.

For what follows, it is convenient to introduce a compact formulation for problem (11). In particular, we define $\mathcal{V}=[v, V]$ a generic function of the space $\mathbb{V}=H_{0}^{1}(\Omega) \times H^{1}(\Lambda)$ and we name $\mathcal{U}=[u, U]$ the couple of

unknowns of problem (11). Any function $\mathcal{V} \in \mathbb{V}$ is endowed with the norm $\|\mathcal{V}\|^{2}=\|v\|_{H^{1}(\Omega)}^{2}+\|V\|_{H^{1}(\Lambda),|\mathcal{D}|}^{2}$. Then, we introduce the following bilinear form in $\mathbb{V} \times \mathbb{V}$,

$$
\mathcal{A}(\mathcal{U}, \mathcal{V})=a_{\Omega}(u, v)+a_{\Lambda}(U, V)+b_{\Lambda}^{\epsilon}(\bar{u}-U, \bar{v}-V),
$$

and the linear functional in $\mathbb{V}, \mathcal{F}(\mathcal{V})=(f, v)_{\Omega}+(\overline{\bar{g}}, V)_{\Lambda,|\mathcal{D}|}$. Then, the compact form of problem (11) consists of finding $\mathcal{U} \in \mathbb{V}$ such that

$$
\mathcal{A}(\mathcal{U}, \mathcal{V})=\mathcal{F}(\mathcal{V}), \quad \forall \mathcal{V} \in \mathbb{V}
$$

\subsection{Well-posedness analysis}

The mathematical properties of problem (12) are studied below. Before proceeding, we list the main assumptions on the domain and on the coefficients, at the basis of the existence of a solution (E1-E3). From now on, the dependence of $\mathcal{D}$ and $\partial \mathcal{D}$ from the arclength $s$ will be omitted in the notation.

E1: $\Lambda \subset \subset \Omega$ are bounded and domains in $\mathbb{R}^{d-2}$ and $\mathbb{R}^{d}$ respectively, with $d=3$.

E2: The domain $\Sigma$ is a generalized cylinder with strictly positive minimal diameter $\min _{s}|\mathcal{D}(s)|>0$ for any $s \in(0, S)$. As a consequence $\Sigma$ has planar faces $|\mathcal{D}(0)|,|\mathcal{D}(S)|>0$ at the endpoints of $\Lambda$.

E3: There exist positive constants $C_{\mathcal{D}}, C_{\partial \mathcal{D}}$ independent of $s$, such that

$$
|\mathcal{D}|=C_{\mathcal{D}}(\operatorname{diam}(\mathcal{D}))^{2}, \quad|\partial \mathcal{D}|=C_{\partial \mathcal{D}} \operatorname{diam}(\mathcal{D}) .
$$

E4: The parameter $\kappa \in L^{\infty}(\Lambda)$ is strictly positive and lower bounded by $\kappa_{\min }>0$.

Remark 3.1. Assumption E2 prevents that the generalized cylinder $\Sigma$ pinches at any interior or extremal location of the centerline $\Lambda$, i.e. the cross section of the cylinder can not collapse. In particular, this entails that the cylinder must have blunt (planar) tips. This is an essential requirement in the analysis, because the boundedness of the bilinear form depends of the minimal radius of the cylinder, as shown in Lemma 3.7. This is due to the fact that, if a cross section collapses, then the coupling between the 3D and the 1D problem becomes ill posed. This is equivalent to observe that, if $|\mathcal{D}| \rightarrow 0$, then we loose control of $U$ in the weighted norm $\|\mathcal{U}\|$. 
Remark 3.2. Assumption E3, namely that $C_{\mathcal{D}}$ and $C_{\partial \mathcal{D}}$ are independent of $s$ means that the cross section of $\Sigma$ can not change its shape, but it can be subject to a homothetic map and rotation.

Theorem 3.3. Under the assumptions E1-E4, problem (12) has a unique solution $\mathcal{U} \in \mathbb{V}$ satisfying the following stability estimate,

with

$$
\|\mathcal{U}\| \leq C_{S 1}\left(\|f\|_{L^{2}(\Omega)}+\|\overline{\bar{g}}\|_{L^{2}(\Lambda),|\mathcal{D}|}\right)
$$

$$
C_{S 1}=\frac{2 \sqrt{1+\left(1+C_{P}(\Omega)\right)^{2} \bar{C}_{T}^{2}\|\kappa\|_{L^{\infty}}^{2}}}{\min \left(\bar{C}_{T}\|\kappa\|_{L^{\infty}}, 2\left(1+\left(1+C_{P}(\Omega)\right) \bar{C}_{T}\|\kappa\|_{L^{\infty}}\right), C_{\partial \mathcal{D}} / C_{\mathcal{D}} \kappa_{\text {min }}\right)},
$$

where the meaning of constants will be clarified in what follows.

Before addressing the central result, we present some auxiliary tools that will be useful in the analysis.

Lemma 3.4. If $v \in H^{1}(\Omega)$ or alternatively $v \in L^{2}(\Gamma)$, then $\bar{v} \in L^{2}(\Lambda)$ and the following inequality holds

$$
\|\bar{v}\|_{L^{2}(\Lambda),|\partial \mathcal{D}|}^{2} \leq\|v\|_{L^{2}(\Gamma)}^{2} \leq C_{T}(\Gamma, \Omega)\|v\|_{H^{1}(\Omega)}^{2},
$$

being $C_{T}(\Gamma, \Omega)$ the (positive) constant of the trace inequality from $L^{2}(\Gamma)$ to $H^{1}(\Omega)$. Moreover, $C_{T}(\Gamma, \Omega)=$ $\mathcal{O}\left(\epsilon^{1-\frac{1}{2-\delta}}\right)$, for $\delta>0$ sufficiently small, therefore it tends to 0 when $\epsilon$ tends to 0 .

Proof. If the inequality (15) holds, it follows immediately that $\bar{v} \in L^{2}(\Lambda)$, since $v \in H^{1}(\Omega)$, or alternatively $v \in L^{2}(\Gamma)$. Therefore, we consider

$$
\|\bar{v}\|_{L^{2}(\Lambda),|\partial \mathcal{D}|}^{2}=\int_{\Lambda}|\partial \mathcal{D}| \bar{v}^{2} d s=\int_{\Lambda} \frac{1}{|\partial \mathcal{D}|}\left(\int_{\partial \mathcal{D}} v d \gamma\right)^{2} d s
$$

Using Jensen's inequality, we obtain

$$
\int_{\Lambda} \frac{1}{|\partial \mathcal{D}|}\left(\int_{\partial \mathcal{D}} v d \gamma\right)^{2} d s \leq \int_{\Lambda} \int_{\partial \mathcal{D}} v^{2} d \gamma d s
$$

and consequently

$$
\|\bar{v}\|_{L^{2}(\Lambda),|\partial \mathcal{D}|}^{2} \leq \int_{\Lambda} \int_{\partial \mathcal{D}} v^{2} d \gamma d s=\|v\|_{L^{2}(\Gamma)}^{2} \leq C_{T}(\Gamma, \Omega)\|v\|_{H^{1}(\Omega)}^{2} .
$$

We now prove that the constant $C_{T}(\Gamma, \Omega)$ tends to 0 when $\epsilon$ tends to 0 analyzing the behaviour of the trace constrant $C\left(\Gamma, \Omega_{\oplus}\right)$ from $L^{2}(\Gamma)$ to $H^{1}\left(\Omega_{\oplus}\right)$ and exploiting the fact that for any function $v \in H^{1}(\Omega)$ we have

$$
\|v\|_{L^{2}(\Gamma)}^{2} \leq C_{T}\left(\Gamma, \Omega_{\oplus}\right)\|v\|_{H^{1}\left(\Omega_{\oplus}\right)}^{2} \leq C_{T}\left(\Gamma, \Omega_{\oplus}\right)\|v\|_{H^{1}(\Omega)}^{2} .
$$

Therefore, let $v \in H^{1}\left(\Omega_{\oplus}\right)$, then the trace of $v$ on $\Gamma$ is in $H^{\frac{1}{2}}(\Gamma)$ and by Sobolev embedding theorem we have that $H^{\frac{1}{2}}(\Gamma)$ is embedded in $L^{p}(\Gamma)$ for $p \leq 4$. Using Hölder inequality with positive exponents $2-\delta$ and $\frac{2-\delta}{1-\delta}$, for $\delta>0$ sufficiently small,

$$
\begin{aligned}
\|v\|_{L^{2}(\Gamma)}^{2} & =\int_{\Gamma} v^{2} d \sigma \leq\left(\int_{\Gamma} 1^{\frac{2-\delta}{1-\delta}} d \sigma\right)^{1-\frac{1}{2-\delta}}\left(\int_{\Gamma} v^{4-2 \delta}\right)^{\frac{1}{2-\delta}} \leq|\Gamma|^{1-\frac{1}{2-\delta}}\|v\|_{L^{4-2 \delta}(\Gamma)}^{2} \\
& \leq|\Gamma|^{1-\frac{1}{2-\delta}} C_{T}\left(\partial \Omega_{\oplus}, \Omega_{\oplus}\right)\|v\|_{H^{1}\left(\Omega_{\oplus}\right)}^{2}
\end{aligned}
$$

where $C_{T}\left(\partial \Omega_{\oplus}, \Omega_{\oplus}\right)$ is bounded as $\epsilon \rightarrow 0$. Indeed, the problem of studying the asymptotic behaviour of the constant in the trace inequality from $L^{4-2 \delta}\left(\partial \Omega_{\oplus}\right)$ to $H^{1}\left(\Omega_{\oplus}\right)$ as $\epsilon \rightarrow 0$ can be reformulated as the problem of studying the behaviour of the trace constant as the external boundary $\partial \Omega$ expands (we recall that $\left.\partial \Omega=\partial \Omega_{\oplus} \backslash \Gamma\right)$. Then, from [16, Theorem 1.3, item 2], taking $p=2, q=4-2 \delta$, we have that $C_{T}\left(\partial \Omega_{\oplus}, \Omega_{\oplus}\right)$ is uniformly bounded with respect to the size of the domain and the relative size of the boundary. Therefore $C_{T}\left(\Gamma, \Omega_{\oplus}\right) \leq|\Gamma|^{1-\frac{1}{2-\delta}} C_{T}\left(\partial \Omega_{\oplus}, \Omega_{\oplus}\right)=\mathcal{O}\left(\epsilon^{1-\frac{1}{2-\delta}}\right)$ when $\epsilon \rightarrow 0$, because the surface area of $\Gamma$ is proportional to $\epsilon$. 
Lemma 3.5 (Poincaré inequality). For any $v \in H_{0}^{1}(\Omega)$, there exists a positive constant, $C_{P}(\Omega)$, s.t.

$$
\|v\|_{L^{2}(\Omega)}^{2} \leq C_{P}(\Omega)\|\nabla v\|_{L^{2}(\Omega)}^{2} .
$$

We now address the well-posedness of problem (12) on the basis of the theory for linear variational problems in Banach spaces. More precisely we use Theorem 2.6 of [15] (also named the Banach-NecasBabuska Theorem), which for the sake of clarity is adapted here to the notation used for (12).

Theorem 3.6. Let $\mathbb{V}$ be a reflexive Banach space; let $\mathcal{A}(\cdot, \cdot)$ be a bounded bilinear form on $\mathbb{V} \times \mathbb{V}$; let $\mathcal{F}$ be a bounded linear functional on $\mathbb{V}$, i.e. $\mathcal{F} \in \mathbb{V}^{\prime}$. Problem (12) is well-posed if and only if:

$$
\begin{gathered}
\exists \alpha>0: \inf _{\mathcal{W} \in \mathbb{V}} \sup _{\mathcal{V} \in \mathbb{V}} \frac{\mathcal{A}(\mathcal{W}, \mathcal{V})}{\|\mathcal{W}\|\|\| \mathcal{V} \|} \geq \alpha, \\
\forall \mathcal{V} \in \mathbb{V}:(\mathcal{A}(\mathcal{W}, \mathcal{V})=0 \forall \mathcal{W} \in \mathbb{V}) \Rightarrow \mathcal{V}=0 .
\end{gathered}
$$

We first analyze the boundedness of the bilinear form and of the functional.

Lemma 3.7. Under the assumptions E1-E4, the bilinear form $\mathcal{A}$ is bounded with respect to the norm $\|\cdot\| \|$,

$$
\mathcal{A}(\mathcal{U}, \mathcal{V}) \leq\|\mathcal{A}\||\|\mathcal{U}\||\|\mathcal{V}\|, \quad \forall \mathcal{U}, \mathcal{V} \in \mathbb{V},
$$

by the constant

$$
\|\mathcal{A}\|=2+\|\kappa\|_{L^{\infty}}\left(\sqrt{C_{T}(\Gamma, \Omega)}+\sqrt{\frac{C_{\partial \mathcal{D}}}{C_{\mathcal{D}} \min _{s}(\operatorname{diam}(\mathcal{D}))}}\right)^{2} .
$$

The functional $\mathcal{F}$ is bounded in $\mathbb{V}$ owing to the inequality,

$$
\mathcal{F}(\mathcal{V}) \leq\left(\|f\|_{L^{2}(\Omega)}+\|\overline{\bar{g}}\|_{L^{2}(\Lambda),|\mathcal{D}|}\right)\|\mathcal{V}\| \|
$$

Proof. We start by recalling the definition of the global bilinear form,

$$
\mathcal{A}(\mathcal{U}, \mathcal{V})=a_{\Omega}(u, v)+a_{\Lambda}(U, V)+b_{\Lambda}^{\epsilon}(\bar{u}-U, \bar{v}-V) .
$$

For the first and the second terms it easily follows that

$$
a_{\Omega}(u, v) \leq\|\mathcal{U}\|\|\| \mathcal{V}\left\|, \quad a_{\Lambda}(U, V) \leq\right\| \mathcal{U}\|\|\|\mathcal{V}\| .
$$

For the last term we have

$$
\begin{aligned}
b_{\Lambda}^{\epsilon}(\bar{u}-U, \bar{v}-V)=(\kappa(\bar{u}-U) & , \bar{v}-V)_{\Lambda,|\partial \mathcal{D}|} \leq\|\kappa\|_{L^{\infty}}\|\bar{u}-U\|_{L^{2}(\Lambda),|\partial \mathcal{D}|}\|\bar{v}-V\|_{L^{2}(\Lambda),|\partial \mathcal{D}|} \\
& \leq\|\kappa\|_{L^{\infty}}\left(\|\bar{u}\|_{L^{2}(\Lambda),|\partial \mathcal{D}|}+\|U\|_{L^{2}(\Lambda),|\partial \mathcal{D}|}\right)\left(\|\bar{v}\|_{L^{2}(\Lambda),|\partial \mathcal{D}|}+\|V\|_{L^{2}(\Lambda),|\partial \mathcal{D}|}\right) .
\end{aligned}
$$

We notice that, using (13), $\forall V \in H^{1}(\Lambda)$

$$
\begin{aligned}
\|V\|_{L^{2}(\Lambda),|\partial \mathcal{D}|}^{2} & =\int_{\Lambda} \frac{|\partial \mathcal{D}|}{|\mathcal{D}|}|\mathcal{D}| V^{2} d s=\frac{C_{\partial \mathcal{D}}}{C_{\mathcal{D}}} \int_{\Lambda} \frac{1}{\operatorname{diam}(\mathcal{D})}|\mathcal{D}| V^{2} d s \\
& \leq \frac{C_{\partial \mathcal{D}}}{C_{\mathcal{D}} \min _{s}(\operatorname{diam}(\mathcal{D}))}\|V\|_{L^{2}(\Lambda),|\mathcal{D}|}^{2} \leq \frac{C_{\partial \mathcal{D}}}{C_{\mathcal{D}} \min _{s}(\operatorname{diam}(\mathcal{D}))}\|V\|_{H^{1}(\Lambda),|\mathcal{D}|}^{2} .
\end{aligned}
$$

Consequently, using Lemma 3.4,

$$
\begin{aligned}
\|\bar{v}\|_{L^{2}(\Lambda),|\partial \mathcal{D}|}+\|V\|_{L^{2}(\Lambda),|\partial \mathcal{D}|} & \leq \sqrt{C_{T}(\Gamma, \Omega)}\|v\|_{H^{1}(\Omega)}+\sqrt{\frac{C_{\partial \mathcal{D}}}{C_{\mathcal{D}} \min _{s}(\operatorname{diam}(\mathcal{D}))}}\|V\|_{H^{1}(\Lambda),|\mathcal{D}|} \\
& \leq\left(\sqrt{C_{T}(\Gamma, \Omega)}+\sqrt{\frac{C_{\partial \mathcal{D}}}{C_{\mathcal{D}} \min _{s}(\operatorname{diam}(\mathcal{D}))}}\right)\|\mathcal{V}\| .
\end{aligned}
$$


Therefore we have,

$$
b_{\Lambda}^{\epsilon}(\bar{u}-U, \bar{v}-V) \leq\|\kappa\|_{L^{\infty}}\left(\sqrt{C_{T}(\Gamma, \Omega)}+\sqrt{\frac{C_{\partial \mathcal{D}}}{C_{\mathcal{D}} \min _{s}(\operatorname{diam}(\mathcal{D}))}}\right)^{2}\|\mathcal{U}\|\|\| \mathcal{V} \|
$$

The boundedness of $\mathcal{F}$ is easily shown owing to Cauchy-Schwarz inequality,

$\mathcal{F}(\mathcal{V})=(f, v)_{\Omega}+(\overline{\bar{g}}, V)_{\Lambda,|\mathcal{D}|} \leq\|f\|_{L^{2}(\Omega)}\|v\|_{L^{2}(\Omega)}+\|\overline{\bar{g}}\|_{L^{2}(\Lambda),|\mathcal{D}|}\|V\|_{L^{2}(\Lambda),|\mathcal{D}|} \leq\left(\|f\|_{L^{2}(\Omega)}+\|\overline{\bar{g}}\|_{L^{2}(\Lambda),|\mathcal{D}|}\right)\|\mathcal{V}\| \mid$

Lemma 3.8. Under the assumptions E1-E4, the operator $\mathcal{A}$ satisfies (BNB1)-(BNB2) with a constant $\alpha$ independent of $\epsilon$.

Proof. In order to prove that the bilinear form $\mathcal{A}$ satisfies (BNB1) it is sufficient to prove that there exists a positive constant $\alpha$ such that $\forall \mathcal{W} \in \mathbb{V}$ we can find $\mathcal{V} \in \mathbb{V}$ satisfying

$$
\frac{\mathcal{A}(\mathcal{W}, \mathcal{V})}{\|\mathcal{W}\| \mid\|\mathcal{V}\|} \geq \alpha
$$

We subdivide the proof in the following steps. We prove that:

(i) $\exists m_{1}, m_{2}, m_{3}>0$ :

$$
\mathcal{A}(\mathcal{V}, \mathcal{V}) \geq m_{1}\|v\|_{H^{1}(\Omega)}^{2}+m_{2}|V|_{H^{1}(\Lambda),|\mathcal{D}|}^{2}+m_{3}\|\bar{v}-V\|_{L^{2}(\Lambda),|\partial \mathcal{D}|}^{2}, \quad \forall \mathcal{V} \in \mathbb{V}
$$

(ii) $\forall \mathcal{W} \in \mathbb{V}, \exists \mathcal{V} \in \mathbb{V}$ and $\alpha_{1}>0$ :

$$
\mathcal{A}(\mathcal{W}, \mathcal{V}) \geq \alpha_{1}\|\mathcal{W}\|^{2}
$$

(iii) and $\exists \alpha_{2}>0$ :

$$
\|\mathcal{W}\|\left\|\alpha_{2}\right\| \mathcal{V} \|
$$

From the last two inequalities we obtain that (BNB1) holds for $\alpha=\alpha_{1} \alpha_{2}$. In details:

(i) By definition of $\mathcal{A}$,

$$
\mathcal{A}(\mathcal{V}, \mathcal{V})=a_{\Omega}(v, v)+a_{\Lambda}(V, V)+b_{\Lambda}^{\epsilon}(\bar{v}-V, \bar{v}-V)
$$

and for the first term we have

$$
a_{\Omega}(v, v)=(\nabla v, \nabla v)_{\Omega} \geq\left(1+C_{P}(\Omega)\right)^{-1}\|v\|_{H^{1}(\Omega)}^{2},
$$

where $C_{P}(\Omega)$ is the Poincaré constant. For the second term we have,

$$
a_{\Lambda}(V, V)=|V|_{H^{1}(\Lambda),|\mathcal{D}|}^{2}
$$

Finally, for the last one we have

$$
b_{\Lambda}^{\epsilon}(\bar{v}-V, \bar{v}-V)=(\kappa(\bar{v}-V), \bar{v}-V)_{\Lambda,|\partial \mathcal{D}|} \geq \kappa_{m i n}\|\bar{v}-V\|_{L^{2}(\Lambda), \partial \mathcal{D}}^{2}
$$

Therefore $(21)$ holds and $m_{1}=\left(1+C_{P}(\Omega)\right)^{-1}, m_{2}=1, m_{3}=\kappa_{\text {min }}$. 
(ii) For any $\mathcal{W}=[w, W]$, we choose $\mathcal{V}=\mathcal{W}+\delta[0, W]$ and from (i) we have

$$
\begin{aligned}
\mathcal{A}(\mathcal{W}, \mathcal{W}+\delta[0, W])= & \mathcal{A}(\mathcal{W}, \mathcal{W})+\delta \mathcal{A}(\mathcal{W},[0, W]) \\
\geq & m_{1}\|w\|_{H^{1}(\Omega)}^{2}+m_{2}|W|_{H^{1}(\Lambda),|\mathcal{D}|}^{2}+m_{3}\|\bar{w}-W\|_{L^{2}(\Lambda),|\partial \mathcal{D}|}^{2} \\
& +\delta\left(a_{\Omega}(w, 0)+a_{\Lambda}(W, W)+b_{\Lambda}^{\epsilon}(\bar{w}-W,-W)\right. \\
\geq & m_{1}\|w\|_{H^{1}(\Omega)}^{2}+m_{2}|W|_{H^{1}(\Lambda),|\mathcal{D}|}^{2} \\
& +\delta\left(|W|_{H^{1}(\Lambda),|\mathcal{D}|}^{2}+(\kappa(\bar{w}-W),-W)_{\Lambda,|\partial \mathcal{D}|}\right) \\
\geq & m_{1}\|w\|_{H^{1}(\Omega)}^{2}+\left(m_{2}+\delta\right)|W|_{H^{1}(\Lambda),|\mathcal{D}|}^{2} \\
& -\delta\left((\kappa \bar{w}, W)_{\Lambda,|\partial \mathcal{D}|}-(\kappa W, W)_{\Lambda,|\partial \mathcal{D}|}\right) .
\end{aligned}
$$

We notice that using Young inequality and Lemma 3.4 we obtain

$$
\begin{aligned}
(\kappa \bar{w}, W)_{\Lambda,|\partial \mathcal{D}|} & =\int_{\Lambda}|\partial \mathcal{D}| \kappa \bar{w} W d s \leq \frac{1}{2}\left(\int_{\Lambda}|\partial \mathcal{D}| \kappa \bar{w}^{2} d s+\int_{\Lambda}|\partial \mathcal{D}| \kappa W^{2} d s\right) \\
& \leq \frac{1}{2}\left(\|\kappa\|_{L^{\infty}}\|\bar{w}\|_{L^{2}(\Lambda),|\partial \mathcal{D}|}^{2}+(\kappa W, W)_{\Lambda,|\partial \mathcal{D}|}\right) \\
& \leq \frac{1}{2}\left(C_{T}(\Gamma, \Omega)\|\kappa\|_{L^{\infty}}\|w\|_{H^{1}(\Omega)}^{2}+(\kappa W, W)_{\Lambda,|\partial \mathcal{D}|}\right) .
\end{aligned}
$$

As a consequence of Lemma (3.4), there exists an upper bound $\bar{C}_{T}$ for the trace constant $C_{T}(\Gamma, \Omega)$ independent of $\epsilon$. Therefore, we have

$$
(\kappa \bar{w}, W)_{\Lambda,|\partial \mathcal{D}|} \leq \frac{1}{2}\left(\bar{C}_{T}\|\kappa\|_{L^{\infty}}\|w\|_{H^{1}(\Omega)}^{2}+(\kappa W, W)_{\Lambda,|\partial \mathcal{D}|}\right) .
$$

Substituting in (24) we have,

$$
\mathcal{A}(\mathcal{W}, \mathcal{W}+\delta[0, W]) \geq\left(m_{1}-\frac{\delta}{2} \bar{C}_{T}\|\kappa\|_{L^{\infty}}\right)\|w\|_{H^{1}(\Omega)}^{2}+\left(m_{2}+\delta\right)|W|_{H^{1}(\Lambda),|\mathcal{D}|}^{2}+\frac{\delta}{2}(\kappa W, W)_{\Lambda,|\partial \mathcal{D}|}
$$

and we choose $\delta=\frac{m_{1}}{\bar{C}_{T}\|\kappa\|_{L^{\infty}}}$ so that $m_{1}-\frac{\delta}{2} \bar{C}_{T}\|\kappa\|_{L^{\infty}}=\frac{m_{1}}{2}$ and it is positive. Using assumption (13) and reminding that $\operatorname{diam}(\mathcal{D}) \leq 1$, we have,

$$
(\kappa W, W)_{\Lambda,|\partial \mathcal{D}|} \geq \kappa_{\min } \int_{\Lambda}|\partial \mathcal{D}| W^{2} d s=\kappa_{\min } \int_{\Lambda} \frac{C_{\partial \mathcal{D}}}{C_{\mathcal{D}} \operatorname{diam}(\mathcal{D})}|\mathcal{D}| W^{2} d s \geq \frac{C_{\partial \mathcal{D}}}{C_{\mathcal{D}}} \kappa_{\min }\|W\|_{L^{2}(\Lambda),|\mathcal{D}|}^{2} .
$$

Therefore

$$
\begin{aligned}
\mathcal{A}(\mathcal{W}, \mathcal{W}+\delta[0, W]) \geq & \frac{m_{1}}{2}\|w\|_{H^{1}(\Omega)}^{2} \\
& +\left(m_{2}+\delta\right)|W|_{H^{1}(\Lambda),|\mathcal{D}|}^{2}+\frac{\delta}{2} \frac{C_{\partial \mathcal{D}}}{C_{\mathcal{D}}} \kappa_{m i n}\|W\|_{L^{2}(\Lambda),|\mathcal{D}|}^{2} \\
\geq & \frac{m_{1}}{2}\|w\|_{H^{1}(\Omega)}^{2}+\min \left(m_{2}+\delta, \frac{\delta}{2} \frac{C_{\partial \mathcal{D}}}{C_{\mathcal{D}}} \kappa_{m i n}\right)\|W\|_{H^{1}(\Lambda),|\mathcal{D}|}^{2} \\
\geq & \alpha_{1}\|\mathcal{W}\|^{2}
\end{aligned}
$$

with $\alpha_{1}=\min \left(\frac{m_{1}}{2}, m_{2}+\delta, \frac{\delta}{2} \frac{C_{\partial \mathcal{D}}}{C_{\mathcal{D}}} \kappa_{\text {min }}\right)=\min \left(\frac{1}{2\left(1+C_{P}(\Omega)\right)}, 1+\frac{1}{\left(1+C_{P}(\Omega)\right) C_{T}\|\kappa\|_{L} \infty}, \frac{1}{2} \frac{C_{\partial \mathcal{D}} \kappa_{\min }}{C_{\mathcal{D}}\left(1+C_{P}(\Omega)\right) C_{T}\|\kappa\|_{L^{\infty}}}\right)$.

(iii) We show that there exists a constant $\alpha_{2}$ such that

$$
\|\mathcal{W}\|\left\|\alpha_{2}\right\| \mathcal{W}+\delta[0, W] \| .
$$


The inequality above can be proved as follows:

$$
\|\mathcal{W}+\delta[0, W]\|^{2} \leq\|\mathcal{W}\|^{2}+\delta^{2}\|[0, W]\|^{2}=\|w\|_{H^{1}(\Omega)}^{2}+\left(1+\delta^{2}\right)\|W\|_{H^{1}(\Lambda),|\mathcal{D}|}^{2} \leq\left(1+\delta^{2}\right)\|\mathcal{W}\|^{2} .
$$

Therefore (26) holds with $\alpha_{2}=\left(\sqrt{1+\delta^{2}}\right)^{-1}=\left(\sqrt{1+\left(\frac{1}{\left(1+C_{P}(\Omega)\right) C_{T}\|\kappa\|_{L} \infty}\right)^{2}}\right)^{-1}$. Being $\alpha_{1}$ and $\alpha_{2}$ independent of $\epsilon$, it follows that also $\alpha$ is independent of $\epsilon$.

For the proof of (BNB2) we choose $\mathcal{W}=\mathcal{V}$ and being $\mathcal{A}(\mathcal{V}, \mathcal{V})=0$, from (i) we have

$$
m_{1}\|v\|_{H^{1}(\Omega)}^{2}+m_{2}|V|_{H^{1}(\Lambda),|\mathcal{D}|}^{2}+m_{3}\|\bar{v}-V\|_{L^{2}(\Lambda),|\partial \mathcal{D}|}^{2}=0,
$$

and consequently

$$
\|v\|_{H^{1}(\Omega)}=0,|V|_{H^{1}(\Lambda),|\mathcal{D}|}=0,\|\bar{v}-V\|_{L^{2}(\Lambda),|\partial \mathcal{D}|}=0 .
$$

Then, $v=0$ and $|V|_{H^{1}(\Lambda),|\mathcal{D}|}=0$ with $\|V\|_{L^{2}(\Lambda),|\partial \mathcal{D}|}^{2}=0$ imply $V=0$.

Combining Lemma 3.8 and Theorem 3.6, we obtain the well-posedness of (12). In order complete the proof of Theorem 3.3, it remains to show that the stability estimate (14) holds. Hence,

$$
\|\mathcal{U}\| \leq \frac{1}{\alpha} \sup _{\mathcal{V} \in \mathbb{V}} \frac{\mathcal{A}(\mathcal{U}, \mathcal{V})}{\|\mid \mathcal{V}\|}=\frac{1}{\alpha} \sup _{\mathcal{V} \in \mathbb{V}} \frac{\mathcal{F}(\mathcal{V})}{\|\mathcal{V}\|} \leq \frac{1}{\alpha}\left(\|f\|_{L^{2}(\Omega)}+\|\overline{\bar{g}}\|_{L^{2}(\Lambda),|\mathcal{D}|}\right)
$$

where the last inequality follows from Lemma 3.7.

\subsection{Additional regularity of the solution of the problem in $\Omega$}

We observe that the weak formulation (11a) could have been formally written in strong form as

$$
-\Delta u=f-\kappa \mathcal{E}_{\Gamma}(\bar{u}-U) \delta_{\Gamma} \text { in } \Omega, \quad u=0 \text { on } \partial \Omega,
$$

where $\delta_{\Gamma}$ is the Dirac measure of the surface $\Gamma$ and $\mathcal{E}_{\Gamma}$ denotes an extension operator from $\Lambda$ to $\Gamma$. More precisely, given a continuous function $\varphi \in C^{0}(\Lambda)$, for any $s \in(0, S)$ the extension operator is such that

$$
\mathcal{E}_{\Gamma} \varphi(r, t ; s)=\varphi(s) \quad \forall r \in(0, R), t \in(0, T),
$$

namely, the extension operator spans the point-wise value $\varphi(s)$ on $\{\boldsymbol{\lambda}(s)+\partial \mathcal{D}(s)\}$, preserving the regularity of the function. It is straightforward to show that $\left(\kappa \mathcal{E}_{\Gamma}(\bar{u}-U) \delta_{\Gamma}, v\right)_{\Omega}$ becomes $b_{\Lambda}^{\epsilon}(\bar{u}-U, \bar{v})$ in the variational formulation, as follows

$$
\int_{\Omega} \kappa \mathcal{E}_{\Gamma}(\bar{u}-U) v \delta_{\Gamma} d \Omega=\int_{\Gamma} \kappa \mathcal{E}_{\Gamma}(\bar{u}-U) v d \sigma=\int_{\Lambda} \kappa(\bar{u}-U) \int_{\partial \mathcal{D}(s)} v d \gamma d s=\int_{\Lambda}|\partial \mathcal{D}| \kappa(\bar{u}-U) \bar{v} d s
$$

Due to the presence of the Dirac source $\delta_{\Gamma}$, global $H^{2}$-regularity can not be recovered and the question arises to which interspace $X$ with $H^{2}(\Omega) \subset X \subset H_{0}^{1}(\Omega)$ the solution $u$ belongs to. This property will be addressed in Theorem 3.11. Our analysis is based on the following regularity properties of elliptic equations studied in [22]. More precisely, we introduce two auxiliary Lemmas, which in turn require some additional assumption on the regularity of domains, listed below.

Lemma 3.9. Let $\Omega$ be a generic bounded, convex domain in $\mathbb{R}^{d}$. Let $\gamma \subset \Omega$ be a $\mathcal{C}^{2}$-surface such that the distance between $\gamma$ and $\partial \Omega$ is positive and $\gamma \subset \partial D$ for some three-dimensional $\mathcal{C}^{2}$-domain $D \subset \subset \Omega$. Consider the following problem

$$
\begin{cases}-\Delta y=\phi \delta_{\gamma} & \text { in } \Omega \\ y=0 & \text { on } \partial \Omega\end{cases}
$$


with $\delta_{\gamma}$ being the Dirac measure of $\gamma$ and $\phi(x) \in L^{2}(\gamma)$. Problem (29) has a unique solution $y$ and $y \in$ $H^{\frac{3}{2}-\eta}(\Omega) \cap H_{0}^{1}(\Omega)$ for each $\eta>0$. Furthermore the function $y$, suitably extended to $\mathbb{R}^{d}$, can be decomposed as

respectively solutions of

$$
y=z+w \quad \text { with } z \in H^{2}(\Omega), w \in H^{\frac{3}{2}-\eta}\left(\mathbb{R}^{d}\right)
$$

$$
\begin{aligned}
& \begin{cases}-\Delta z=-y \Delta(1-F)-2 \nabla(1-F) \nabla y & \text { in } \Omega, \\
z=0 & \text { on } \partial \Omega,\end{cases} \\
& -\Delta w=-y \Delta F-2 \nabla F \nabla y+\phi \delta_{\gamma} \quad \text { in } \mathbb{R}^{d},
\end{aligned}
$$

where $F$ is a regular function such that $-y \Delta(1-F)-2 \nabla(1-F) \nabla y \in L^{2}(\Omega)$ and $-y \Delta F-2 \nabla F \nabla y \in L^{2}\left(\mathbb{R}^{d}\right)$.

Proof. For the proof see [22, Theorem 2.1, case (iii)].

Lemma 3.10. Under the assumptions of Lemma 3.9 and the additional assumption that $\Omega$ has a $\mathcal{C}^{2}$ boundary $\partial \Omega$, problem (29) satisfies the following stability estimate in $H^{\frac{3}{2}-\eta}(\Omega)$ : there exists a positive constant $C_{R}$ such that

$$
\|y\|_{H^{\frac{3}{2}-\eta}(\Omega)} \leq C_{R}\left\|\phi \delta_{\gamma}\right\|_{H^{-\frac{1}{2}-\eta}(\Omega)} .
$$

Proof. From the decomposition of Lemma 3.9 we have

$$
\|y\|_{H^{\frac{3}{2}-\eta}(\Omega)} \leq\|z\|_{H^{2}(\Omega)}+\|w\|_{H^{\frac{3}{2}-\eta}\left(\mathbb{R}^{d}\right)} .
$$

For the sake of simplicity, we will denote the positive constants appearing in the following inequalities always with the simbol $C_{R}$, even if they might have different meanings and values. Being $\partial \Omega$ of class $\mathcal{C}^{2}$, since $z \in H_{0}^{1}(\Omega)$, from [20, Theorem 8.12] we obtain that there exists a constant $C_{R}$ such that

$$
\|z\|_{H^{2}(\Omega)} \leq C_{R}\left(\|z\|_{L^{2}(\Omega)}+\|y \Delta(1-F)+2 \nabla(1-F) \nabla y\|_{L^{2}(\Omega)}\right) .
$$

Then, owing to the standard $H^{1}$-stability of the Poisson problem with right-hand side in $L^{2}(\Omega)$, we obtain

$$
\|z\|_{H^{2}(\Omega)} \leq C_{R}\|y \Delta(1-F)+2 \nabla(1-F) \nabla y\|_{L^{2}(\Omega)} \leq C_{R}\|y\|_{H^{1}(\Omega)} .
$$

Still exploiting the $H^{1}$-stability of the Poisson problem with $H^{-1}(\Omega)$ right-hand side, reminding that $\phi \delta_{\gamma} \in$ $H^{-\frac{1}{2}-\eta}(\Omega) \subset H^{-1}(\Omega)$, we obtain

and consequently

$$
\|y\|_{H^{1}(\Omega)} \leq C_{R}\left\|\phi \delta_{\gamma}\right\|_{H^{-\frac{1}{2}-\eta}\left(\mathbb{R}^{d}\right)}
$$

$$
\|z\|_{H^{2}(\Omega)} \leq C_{R}\left\|\phi \delta_{\gamma}\right\|_{H^{-\frac{1}{2}-\eta}\left(\mathbb{R}^{d}\right)} .
$$

Concerning the upper bound of the function $w$, we exploit the theory of pseudo-differential operators. More precisely, we use the generalization of the Garding inequality to fractional Sobolev spaces, see in particular Proposition 5.4 of [1] and its consequences, to show that there exists a constant $C_{R}$ such that

$$
\|w\|_{H^{\frac{3}{2}-\eta}\left(\mathbb{R}^{d}\right)} \leq C_{R}\left(\|\Delta w\|_{H^{-\frac{1}{2}-\eta}\left(\mathbb{R}^{d}\right)}+\|w\|_{H^{1}\left(\mathbb{R}^{d}\right)}\right) .
$$

Then we have

$$
\|\Delta w\|_{H^{-\frac{1}{2}-\eta}\left(\mathbb{R}^{d}\right)} \leq\|y \Delta F+2 \nabla F \nabla y\|_{L^{2}(\Omega)}+\left\|\phi \delta_{\gamma}\right\|_{H^{-\frac{1}{2}-\eta}\left(\mathbb{R}^{d}\right)} \leq C_{R}\|y\|_{H^{1}(\Omega)}+\left\|\phi \delta_{\gamma}\right\|_{H^{-\frac{1}{2}-\eta}\left(\mathbb{R}^{d}\right)}
$$

and for the stability of the Poisson problem in $H^{1}\left(\mathbb{R}^{d}\right)$ we obtain

$$
\|w\|_{H^{1}\left(\mathbb{R}^{d}\right)} \leq C_{R}\left(\|y\|_{H^{1}(\Omega)}+\left\|\phi \delta_{\gamma}\right\|_{H^{-\frac{1}{2}-\eta}\left(\mathbb{R}^{d}\right)}\right) .
$$


Therefore,

$$
\begin{aligned}
\|w\|_{H^{\frac{3}{2}-\eta}\left(\mathbb{R}^{d}\right)} & \leq C_{R}\left(\|y\|_{H^{1}(\Omega)}+\left\|\phi \delta_{\gamma}\right\|_{H^{-\frac{1}{2}-\eta}\left(\mathbb{R}^{d}\right)}\right) \\
& \leq C_{R}\left\|\phi \delta_{\gamma}\right\|_{H^{-\frac{1}{2}-\eta}\left(\mathbb{R}^{d}\right)}
\end{aligned}
$$

Putting together all the previous estimates, we obtain (30).

In order to apply Lemma 3.9 to our case, we require that the domains $\Omega$ and $\Sigma$ satisfy the following regularity assumptions (R1-R3):

R1: $\Omega$ is a convex domain in $\mathbb{R}^{d}$ with $C^{2}$-regular boundary $\partial \Omega$.

R2: $\Sigma \in \mathbb{R}^{d}$ has a $C^{2}$-regular boundary $\partial \Sigma$.

R3: $\Sigma$ is completely embedded into $\Omega$, such that the distance between $\partial \Omega$ and $\partial \Sigma$ is strictly positive.

Theorem 3.11. Under the assumptions R1-R3, in addition to those of Theorem 3.3, the sub-problem on $\Omega$ enjoys additional regularity $u \in H^{\frac{3}{2}-\eta}(\Omega)$ for any $\eta>0$ and the following estimate is satisfied,

$$
\|u\|_{H^{\frac{3}{2}-\eta}(\Omega)} \leq C_{S 2}\left(\|f\|_{L^{2}(\Omega)}+\|\overline{\bar{g}}\|_{L^{2}(\Lambda),|\mathcal{D}|}\right)
$$

where

$$
C_{S 2}:=C_{R}\left\{1+2 \max \left(\sqrt{C_{T}(\Gamma, \Omega) C_{T}^{\prime}(\Gamma, \Omega)}, \sqrt{\frac{C_{T}^{\prime}(\Gamma, \Omega) C_{\partial \mathcal{D}}}{C_{\mathcal{D}} \min _{s}(\operatorname{diam}(\mathcal{D}))}}\right) C_{S 1}\right\} .
$$

Proof. The proof of Theorem 3.11 is a direct consequence of Lemmas 3.9 and 3.10. Owing to Theorem 3.3 we have $-\kappa \mathcal{E}_{\Gamma}(\bar{u}-U) \in L^{2}(\Gamma)$, so that Lemma 3.9 can be directly applied to equation (28). For the upper bound, inequality (30) implies that

$$
\|u\|_{H^{\frac{3}{2}-\eta}(\Omega)} \leq C_{R}\left(\|f\|_{L^{2}(\Omega)}+\left\|\mathcal{E}_{\Gamma} \bar{u} \delta_{\Gamma}\right\|_{H^{-\frac{1}{2}-\eta}(\Omega)}+\left\|\mathcal{E}_{\Gamma} U \delta_{\Gamma}\right\|_{H^{-\frac{1}{2}-\eta}(\Omega)}\right) .
$$

From the definition of $\|\cdot\|_{H^{-\frac{1}{2}-\eta}(\Omega)}$, we have

$$
\left\|\mathcal{E}_{\Gamma} \bar{u} \delta_{\Gamma}\right\|_{H^{-\frac{1}{2}-\eta}(\Omega)}=\sup _{\substack{w \in H^{\frac{1}{2}+\eta}(\Omega) \\ w \neq 0}} \frac{\left(\mathcal{E}_{\Gamma} \bar{u}, w\right)_{\Gamma}}{\|w\|_{H^{\frac{1}{2}+\eta}(\Omega)}} .
$$

Then, we apply trace inequalities to derive the following upper bound,

$$
\left(\mathcal{E}_{\Gamma} \bar{u}, w\right)_{\Gamma} \leq\|\bar{u}\|_{L^{2}(\Lambda),|\partial \mathcal{D}|}\|w\|_{L^{2}(\Gamma)} \leq \sqrt{C_{T}(\Gamma, \Omega) C_{T}^{\prime}(\Gamma, \Omega)}\|u\|_{H^{1}(\Omega)}\|w\|_{H^{\frac{1}{2}+\eta}(\Omega)},
$$

where $C_{T}^{\prime}(\Gamma, \Omega)$ is the constant in the trace inequality from $L^{2}(\Gamma)$ to $H^{\frac{1}{2}+\eta}(\Omega)$. Reasoning as in Lemma 3.4 for $C_{T}(\Gamma, \Omega)$, we observe that $C_{T}^{\prime}(\Gamma, \Omega)$ is (at least) upper bounded when $\epsilon$ tends to 0 . Proceeding similarly for the last term of (32) and using (19) we obtain,

$$
\left\|\mathcal{E}_{\Gamma} U \delta_{\Gamma}\right\|_{H^{-\frac{1}{2}-\eta}(\Omega)} \leq \sqrt{C_{T}^{\prime}(\Gamma, \Omega)}\|U\|_{L^{2}(\Lambda),|\partial \mathcal{D}|} \leq \sqrt{\frac{C_{T}^{\prime}(\Gamma, \Omega) C_{\partial \mathcal{D}}}{C_{\mathcal{D}} \min _{s}(\operatorname{diam}(\mathcal{D}))}}\|U\|_{H^{1}(\Lambda),|\mathcal{D}|}
$$

Putting together the previous inequalities we obtain,

$$
\|u\|_{H^{\frac{3}{2}-\eta}(\Omega)} \leq C_{R}\left\{\|f\|_{L^{2}(\Omega)}+2 \max \left[\sqrt{C_{T}(\Gamma, \Omega) C_{T}^{\prime}(\Gamma, \Omega)}, \sqrt{\frac{C_{T}^{\prime}(\Gamma, \Omega) C_{\partial \mathcal{D}}}{C_{\mathcal{D}} \min _{s}(\operatorname{diam}(\mathcal{D}))}}\right]\|\mathcal{U}\|\right\},
$$

that combined with (14) gives the desired result. 


\section{MODEL ERROR ANALYSIS}

We study the error introduced by replacing the model (1) with (11). We recall the a posteriori analysis of modeling error developed in [7] for general abstract problems and we apply it here to the particular case of topological model reduction for small cylindrical inclusions. The main objective is to characterize the dependence of the modeling error on the radius of the inclusion, namely $\epsilon$. To this purpose, we split the modeling error in three components, corresponding the assumptions A1, A2, A3 described in Section 2. Each component will be analyzed in one of the following subsections.

Before proceeding, we recall the abstract theory developed in [7], for the particular case of linear elliptic problems. Let $u$ be the solution of the reduced problem and let $u_{\text {ref }}$ be the one of the reference problem, which are respectively defined as follows:

$$
\begin{gathered}
\text { find } u_{\text {ref }} \in X: a_{\text {ref }}\left(u_{\text {ref }}, v\right)=\mathcal{F}_{\text {ref }}(v), \forall v \in X, \\
\text { find } u \in X: a(u, v)=\mathcal{F}(v), \forall v \in X,
\end{gathered}
$$

where $X$ is a suitable Hilbert space, $X^{\prime}$ its dual space and $a_{\text {ref }}$ and $a$ are the bilinear forms that characterize the reference and reduced problem respectively. We assume that $a_{\text {ref }}$ and $\mathcal{F}_{\text {ref }}$ can be expressed as a modification of the form $a$ and the functional $\mathcal{F}$ as follows

$$
\begin{gathered}
a_{\text {ref }}(u, v)=a(u, v)+d(u, v), \forall u, v \in X, \\
\mathcal{F}_{\text {ref }}(v)=\mathcal{F}(v)+l(v), \quad \forall v \in X,
\end{gathered}
$$

As a result, the modeling error $e=u_{\text {ref }}-u \in X$ satisfies the following equation,

$$
a(e, v)+d\left(u_{\mathrm{ref}}, v\right)=l(v), \forall v \in X .
$$

Let $j(\cdot): X \rightarrow \mathbb{R}$ be a linear functional. We aim to estimate the modeling error measured by $j(e)$ using the dual weighted residual approach. We define the dual problem with respect to (33),

$$
\text { find } z_{\text {ref }} \in X: a_{\text {ref }}\left(v, z_{\text {ref }}\right)=j(v), \forall v \in X \text {. }
$$

Following the approach presented in [7], under the assumptions stated above, we obtain that the error output functional is represented as follows,

$$
j(e)=a_{\mathrm{ref}}\left(e, z_{\mathrm{ref}}\right)=a\left(e, z_{\mathrm{ref}}\right)+d\left(u_{\mathrm{ref}}-u, z_{\mathrm{ref}}\right)=l\left(z_{\mathrm{ref}}\right)-d\left(u, z_{\mathrm{ref}}\right) .
$$

The aim of this section is to analytically characterize the asymptotic behavior of the error when the small parameter $\epsilon \rightarrow 0^{+}$. We introduce two fundamental properties that will be analyzed case by case later on. One is that the bilinear form $d$ and the functional $l$ are bounded in suitable norms $\|\cdot\|_{\star}$ and $\|\cdot\|_{\circ}$. The second property concerns stability estimates for the solutions of the reduced primal and reference dual problems.

Property 4.1. There exist constants $\|d\|_{\star \circ},\|l\|_{\circ}$ such that

$$
d\left(u, z_{\text {ref }}\right) \leq\|d\|_{\star \circ}\|u\|_{\star}\left\|z_{\text {ref }}\right\|_{\circ}, \quad l\left(z_{\text {ref }}\right) \leq\|l\|_{\circ}\left\|z_{\text {ref }}\right\|_{\circ} .
$$

Furthermore, the constants $\|d\|_{\star \circ}$ and $\|l\|_{\circ}$ asymptotically vanish with $\epsilon \rightarrow 0$.

Property 4.2. There exist constants $\|a\|_{\star}$ and $\left\|a_{\mathrm{ref}}\right\|_{\circ}$, uniformly bounded with $\epsilon$, such that

$$
\|u\|_{\star} \leq\|a\|_{\star}\|\mathcal{F}\|_{X^{\prime}}, \quad\left\|z_{\text {ref }}\right\|_{\circ} \leq\left\|a_{\text {ref }}\right\|_{\circ}\|j\|_{X^{\prime}} .
$$

Combining Properties 4.1 and 4.2 we obtain the characterization of the asymptotic behavior of the modeling error with respect to $\epsilon$. We are particularly interested in the case $j(v)=(e, v)_{L^{2}}$, because it allows us to derive the following bound,

$$
\|e\|_{L^{2}}^{2}=j(e)=l\left(z_{\text {ref }}\right)-d\left(u, z_{\text {ref }}\right) \leq\|l\|_{\circ}\left\|z_{\text {ref }}\right\|_{\circ}+\|d\|_{\star \circ}\|u\|_{\star}\left\|z_{\text {ref }}\right\|_{\circ} \leq\left(\|l\|_{\circ}+\|d\|_{\star \circ}\|a\|_{\star}\|\mathcal{F}\|_{X^{\prime}}\right)\left\|a_{\text {ref }}\right\|_{\circ}\|e\|_{L^{2}},
$$


which directly entails the $L^{2}$ control of the modeling error.

We will pursue this analysis for the three sources of error, corresponding to assumptions A1, A2, A3. As a result, we will study three different operators $d^{(k)}(\cdot, \cdot)$ and $l^{(k)}(\cdot)$ with $k=1,2,3$, each one corresponding to a different source of error. Before proceeding, we introduce some results that will be useful in what follows.

Lemma 4.1 (Stekloff inequality [29]). Let $\partial \mathcal{D}$ be an ellipse or a rhombus. There exists a positive constant $C$ such that for any function $v \in H^{1}(\mathcal{D})$ satisfying

$$
\int_{\partial \mathcal{D}} v d \gamma=0
$$

the following inequality holds

$$
\int_{\partial \mathcal{D}} v^{2} d \gamma \leq C \int_{\mathcal{D}}(\nabla v)^{2} d \sigma
$$

More precisely if $\partial \mathcal{D}$ is an ellipse $\left(x_{1} / a_{1}\right)^{2}+\left(x_{2} / a_{2}\right)^{2}=1$ then $C \leq \max \left[a_{1}, a_{2}\right]$. If $\partial \mathcal{D}$ is a rhombus $\left|x_{1} / a_{1}\right|+\left|x_{2} / a_{2}\right|=1$, then $C \leq\left(a_{1}^{2}+a_{2}^{2}\right)^{\frac{1}{2}} / \min \left[a_{1} / a_{2}, a_{2} / a_{1}\right]$.

From the previous lemma combined with assumption A0, we conclude that there exists $C_{S}$, independent of $\epsilon$, such that for any $v \in H^{k}(\Sigma)$, with $k \geq \frac{3}{2}$, satisfying (38), we have

$$
\int_{\Gamma} v^{2} d \sigma \leq C_{S} \epsilon \int_{\Sigma}(\nabla v)^{2} d \omega
$$

Lemma 4.2 (Poincaré-Wirtinger inequality [34]). Let $\mathcal{D} \in \mathbb{R}^{2}$ be a convex domain of diameter $\mathrm{D}$. For any function $v \in H^{1}(\mathcal{D})$ such that

$$
\int_{\mathcal{D}} v d \sigma=0
$$

we have,

$$
\int_{\mathcal{D}} v^{2} d \sigma \leq \frac{\mathrm{D}^{2}}{\pi^{2}} \int_{\mathcal{D}}(\nabla v)^{2} d \sigma
$$

Lemma 4.3 (Extension theorem for domains having small geometric details [41]). Let $s$ be a nonnegative integer. There exists an extension operator $\mathcal{E}_{\Sigma}$ from $H^{s}\left(\Omega_{\oplus}\right)$ to $H^{s}(\Sigma)$ such that

$$
\left\|\mathcal{E}_{\Sigma} v\right\|_{H^{s}(\Sigma)} \leq C_{E}\|v\|_{H^{s}\left(\Omega_{\oplus}\right)} \quad \forall v \in H^{s}\left(\Omega_{\oplus}\right)
$$

where the constant $C_{E}$ is independent of $\Sigma=\Omega \backslash \bar{\Omega}_{\oplus}$.

Lemma 4.4 (Poincaré-Friedrichs inequality $[8,34]$ ). There exists a positive constant $C_{P F}(\Sigma)$ such that for any $v \in H^{1}(\Sigma)$ it holds,

$$
\|v\|_{L^{2}(\Sigma)}^{2} \leq C_{P F}(\Sigma)\left(\|\nabla v\|_{L^{2}(\Sigma)}^{2}+\|v\|_{L^{2}(\Gamma)}^{2}\right)
$$

and $C_{P F}(\Sigma)$ tends to 0 for $\epsilon \rightarrow 0$.

Proof. Integrating by parts we have:

$$
\|v\|_{L^{2}(\Sigma)}^{2}=\frac{1}{2} \sum_{i=1}^{2} \int_{\Lambda} \int_{\mathcal{D}} v^{2} \cdot 1 d \sigma d s=-\frac{1}{2} \sum_{i=1}^{2} \int_{\Lambda} \int_{\mathcal{D}} 2 v \frac{\partial v}{\partial x_{i}} x_{i} d \sigma d s+\frac{1}{2} \sum_{i=1}^{2} \int_{\Lambda} \int_{\partial \mathcal{D}} v^{2} x_{i} n_{i} d \gamma d s
$$


where $x_{1}, x_{2}$ is a generic system of coordinates for $\mathcal{D}$ and $n_{i}$ is the $i$-th component of the outward unit surface normal to $\mathcal{D}$. Then, using Schwarz and Young inequalities we obtain

$$
\begin{aligned}
\|v\|_{L^{2}(\Sigma)}^{2} & \leq \frac{1}{2} \sum_{i=1}^{2} 2 \epsilon\|v\|_{L^{2}(\Sigma)}\left\|\frac{\partial v}{\partial x_{i}}\right\|_{L^{2}(\Sigma)}+\frac{1}{2} \sum_{i=1}^{2} \epsilon\|v\|_{L^{2}(\Gamma)}^{2} \\
& \leq \frac{1}{2}\|v\|_{L^{2}(\Sigma)}^{2}+\frac{1}{2} \sum_{i=1}^{2} 2 \epsilon^{2}\left\|\frac{\partial v}{\partial x_{i}}\right\|_{L^{2}(\Sigma)}^{2}+\epsilon\|v\|_{L^{2}(\Gamma)}^{2} \\
& \leq \frac{1}{2}\|v\|_{L^{2}(\Sigma)}^{2}+\epsilon^{2}\|\nabla v\|_{L^{2}(\Sigma)}^{2}+\epsilon\|v\|_{L^{2}(\Gamma)}^{2}
\end{aligned}
$$

from which it follows that

$$
\|v\|_{L^{2}(\Sigma)}^{2} \leq \max \left(2 \epsilon^{2}, 2 \epsilon\right)\left(\|\nabla v\|_{L^{2}(\Sigma)}^{2}+\|v\|_{L^{2}(\Gamma)}^{2}\right)
$$

Thus, $C_{P F}(\Sigma)=\max \left(2 \epsilon^{2}, 2 \epsilon\right)$ and it tends to 0 when $\epsilon$ tends to 0 .

\subsection{Analysis of the modeling error of the one dimensional problem (assumption A1)}

We aim to study the modeling error of replacing the equation (1b) with (6). Since this analysis refer to the assumption A1, we will endow the general operators of the previous section with the apex (1). The reduced problem (reported here for the sake of clarity) is to find $U \in H^{1}(\Lambda)$ such that

$$
\left(d_{s} U, d_{s} V\right)_{\Lambda,|\mathcal{D}|}+(\kappa U, V)_{\Lambda,|\partial \mathcal{D}|}=\left(\kappa \bar{u}_{\oplus}, V\right)_{\Lambda,|\partial \mathcal{D}|}+(\overline{\bar{g}}, V)_{\Lambda,|\mathcal{D}|} \quad \forall V \in H^{1}(\Lambda) .
$$

where $u_{\oplus}$ is the weak solution of (1) and it belongs to the space of functions of $H^{1}\left(\Omega_{\oplus}\right)$ with null trace on $\partial \Omega$, denoted as $H_{\partial \Omega}^{1}\left(\Omega_{\oplus}\right)$. The reference problem in the weak form consists of finding $u_{\ominus} \in H^{1}(\Sigma)$ such that

$$
\left(\nabla u_{\ominus}, \nabla v\right)_{\Sigma}+\left(\kappa u_{\ominus}, v\right)_{\Gamma}=\left(\kappa u_{\oplus}, v\right)_{\Gamma}+(g, v)_{\Sigma}, \forall v \in H^{1}(\Sigma) .
$$

We define the modeling error $e^{(1)}$ as the difference between $u_{\ominus}$ and $U$. To this purpose, we exploit the cylindrical configuration of the domain $\Sigma$ and its local coordinate system. In particular, we uniformly extend $U(s)$ on every cross section $\mathcal{D}(s)$ of the cylinder and with abuse of notation we still denote the extended function with $U$. Thanks to the regularity of $U$ on $\Lambda$, we have that the extension on $\Sigma$ belongs to $H^{1}(\Sigma)$. Referring to the general notation introduced in the previous section we have that the solutions of the reference and reduced models are $u_{\mathrm{ref}}^{(1)}=u_{\ominus}$ and $u^{(1)}=U$ both in the space $X^{(1)}=H^{1}(\Sigma)$. As a result, the modeling error is $e^{(1)}=u_{\mathrm{ref}}^{(1)}-u^{(1)}=u_{\ominus}-U \in H^{1}(\Sigma)$. The bilinear forms of the reference and reduced problems are

$$
\begin{aligned}
a_{\mathrm{ref}}^{(1)}(u, v) & =(\nabla u, \nabla v)_{\Sigma}+(\kappa u, v)_{\Gamma}, \\
a^{(1)}(u, v) & =\left(d_{s} \overline{\bar{u}}, \overline{\overline{\partial_{s} v}}\right)_{\Lambda,|\mathcal{D}|}+\left(\nu(\overline{\bar{u}}-\bar{u}), \overline{\partial_{s} v}\right)_{\Lambda,|\partial \mathcal{D}|}+(\kappa \bar{u}, \bar{v})_{\Lambda,|\partial \mathcal{D}|}, \\
& =\left(d_{s} \overline{\bar{u}}, \partial_{s} v\right)_{\Sigma}+\left(\nu(\overline{\bar{u}}-\bar{u}), \partial_{s} v\right)_{\Gamma}+(\kappa \bar{u}, v)_{\Gamma},
\end{aligned}
$$

where $a^{(1)}(u, v)$ is a generalization of the bilinear form of the reduced problem that can be applied to any function $u, v \in H^{1}(\Sigma)$. More precisely, it is the generalization to any test function $v \in H^{1}(\Sigma)$ of $(5)$. When applied to $U, V \in H^{1}(\Lambda)$ the bilinear form $a^{(1)}(U, V)$ coincides with (6). We aim to quantify the difference $a_{\text {ref }}^{(1)}(u, v)-a^{(1)}(u, v)$. Using the expression of the gradient in cylindrical coordinates $\nabla(\cdot)=$ $\left[\partial_{s}(\cdot), \partial_{r}(\cdot), r^{-1} \partial_{\theta}(\cdot)\right]^{\prime}$, the difference operator $d^{(1)}(\cdot, \cdot)$ becomes

$$
\begin{aligned}
d^{(1)}(u, v) & =a_{\mathrm{ref}}^{(1)}(u, v)-a^{(1)}(u, v) \\
& =\left(\partial_{s} u-d_{s} \overline{\bar{u}}, \partial_{s} v\right)_{\Sigma}-\left(\nu(\overline{\bar{u}}-\bar{u}), \partial_{s} v\right)_{\Gamma}+(\kappa(u-\bar{u}), v)_{\Gamma}+\left(\partial_{r} u, \partial_{r} v\right)_{\Sigma}+\left(r^{-1} \partial_{\theta} u, r^{-1} \partial_{\theta} v\right)_{\Sigma} .
\end{aligned}
$$


Similarly, the difference $l^{(1)}(\cdot)$ of the right hand sides is

$$
l^{(1)}(v)=((\mathcal{I}-\overline{\overline{(\cdot)}}) g, v)_{\Sigma}+\left(\kappa(\mathcal{I}-\overline{(\cdot)}) u_{\oplus}, v\right)_{\Gamma} .
$$

We now aim to prove properties 36 and 37 for the operators $d^{(1)}, l^{(1)}$ and $a^{(1)}, a_{\text {ref }}^{(1)}$ respectively.

Lemma 4.5. The operator $d^{(1)}(\cdot, \cdot)$ satisfies Property 4.1 with $\left\|d^{(1)}\right\|_{\star \circ}=0$.

Proof. Let $U \in H^{1}(\Sigma)$ be the extension to $\Sigma$ of the reduced problem on $\Lambda$. We observe that $d(U, v)=0$ for any $v \in H^{1}(\Sigma)$, because $\partial_{r} U=\partial_{\theta} U=0$ and $U=\overline{\bar{U}}=\bar{U}$.

Lemma 4.6. Under the assumption that $g \in H^{\frac{3}{2}}(\Sigma)$ and $u_{\oplus} \in H_{\partial \Omega}^{1}\left(\Omega_{\oplus}\right) \cap H^{2}\left(\Omega_{\oplus}\right)$, the operator $l^{(1)}(\cdot)$ satisfies Property 4.1 with the norm $\|\cdot\|_{\circ}^{2}=\|\cdot\|_{L^{2}(\Sigma)}^{2}+\|\cdot\|_{L^{2}(\Gamma)}^{2}$ and with the constant

$$
\left\|l^{(1)}\right\|_{\circ}=\frac{\epsilon}{\pi}\|g\|_{H^{1}(\Sigma)}+\|\kappa\|_{L^{\infty}} C_{E} \sqrt{C_{S} \epsilon}\left\|u_{\oplus}\right\|_{H^{1}\left(\Omega_{\oplus}\right)} .
$$

Proof. For the upper bound of the right hand side, we observe that $g \in H^{\frac{3}{2}}(\Sigma)$ implies that $g_{\mid \mathcal{D}} \in H^{1}(\mathcal{D})$. Then, $(\mathcal{I}-\overline{\overline{(\cdot)}}) g$ satisfies the assumptions of the Poincaré-Wirtinger inequality, which allows us to conclude that

$$
\begin{aligned}
((\mathcal{I}-\overline{\overline{(\cdot)}}) g, v)_{\Sigma} & \leq\left(\int_{\Sigma}((\mathcal{I}-\overline{\overline{(\cdot)}}) g)^{2}\right)^{\frac{1}{2}}\left(\int_{\Sigma} v^{2}\right)^{\frac{1}{2}}=\left(\int_{\Lambda} \int_{\mathcal{D}}((\mathcal{I}-\overline{\overline{(\cdot)}}) g)^{2} d \sigma d s\right)^{\frac{1}{2}}\|v\|_{L^{2}(\Sigma)} \\
& \leq\left(\int_{\Lambda} \frac{(\operatorname{diam}(\mathcal{D}))^{2}}{\pi^{2}} \int_{\mathcal{D}}\left(\nabla_{\mathcal{D}} g\right)^{2} d \sigma d s\right)^{\frac{1}{2}}\|v\|_{L^{2}(\Sigma)} \leq \frac{\epsilon}{\pi}\|g\|_{H^{1}(\Sigma)}\|v\|_{\circ},
\end{aligned}
$$

where, from now on $\nabla_{\mathcal{D}}(\cdot)=\left[\partial_{r}(\cdot), r^{-1} \partial_{\theta}(\cdot)\right]^{\prime}$ denotes the gradient in a local coordinate system of $\mathcal{D}$.

For the second term of $l^{(1)}$ we use the Stekloff inequality,

$$
\begin{aligned}
\left(\kappa(\mathcal{I}-\overline{(\cdot)}) u_{\oplus}, v\right)_{\Gamma} & \left.\leq\|\kappa\|_{L^{\infty}}\left(\int_{\Gamma}(\mathcal{I}-\overline{(\cdot)}) u_{\oplus}\right)^{2}\right)^{\frac{1}{2}}\left(\int_{\Gamma} v^{2}\right)^{\frac{1}{2}} \\
& \leq\|\kappa\|_{L^{\infty}} \sqrt{C_{S} \epsilon}\left(\int_{\Lambda} \int_{\mathcal{D}}\left(\nabla_{\mathcal{D}} \mathcal{E}_{\Sigma} u_{\oplus}\right)^{2} d \sigma d s\right)^{\frac{1}{2}}\|v\|_{L^{2}(\Gamma)} \\
& \leq\|\kappa\|_{L^{\infty}} \sqrt{C_{S} \epsilon}\left\|\mathcal{E}_{\Sigma} u_{\oplus}\right\|_{H^{1}(\Sigma)}\|v\|_{L^{2}(\Gamma)} \leq\|\kappa\|_{L^{\infty}} C_{E} \sqrt{C_{S} \epsilon}\left\|u_{\oplus}\right\|_{H^{1}\left(\Omega_{\oplus}\right)}\|v\|_{\circ},
\end{aligned}
$$

where $\mathcal{E}_{\Sigma}$ denotes the extension operator of Lemma 4.3. The previous inequalities show that (36) is verified for this component of the modeling error with the constant,

$$
\left\|l^{(1)}\right\|_{\circ}=\frac{\epsilon}{\pi}\|g\|_{H^{1}(\Sigma)}+\|\kappa\|_{L^{\infty}} C_{E} \sqrt{C_{S} \epsilon}\left\|u_{\oplus}\right\|_{H^{1}\left(\Omega_{\oplus}\right)} .
$$

Lemma 4.7. The dual problem, that is to find $z_{\text {ref }}^{(1)} \in H^{1}(\Sigma)$ such that

$$
\left(\nabla v, \nabla z_{\mathrm{ref}}^{(1)}\right)_{\Sigma}+\left(\kappa v, z_{\mathrm{ref}}^{(1)}\right)_{\Gamma}=j^{(1)}(v), \forall v \in H^{1}(\Sigma)
$$

with $j^{(1)}(v)=(e, v)_{\Sigma}$, satisfies the following stability estimate with the norm $\|\cdot\|_{\circ}=\|\cdot\|_{L^{2}(\Sigma)}+\|\cdot\|_{L^{2}(\Gamma)}$

$$
\left\|z_{\mathrm{ref}}^{(1)}\right\|_{\circ} \leq \frac{2}{\min \left(2 C_{P F}^{-1}(\Sigma), C_{P F}^{-1}(\Sigma) \kappa_{\min }, \kappa_{\min }\right)}\|e\|_{L^{2}(\Sigma)} .
$$

As a result, Property 4.2 is satisfied with the following constant, which is bounded for $\epsilon \rightarrow 0$,

$$
\left\|a_{\mathrm{ref}}^{(1)}\right\|_{\circ}=\frac{2}{\min \left(2 C_{P F}^{-1}(\Sigma), C_{P F}^{-1}(\Sigma) \kappa_{\min }, \kappa_{\min }\right)} .
$$


Proof. Owing to Poincaré -Friedrichs inequality (41) we have,

$$
\begin{aligned}
a_{\mathrm{ref}}(v, v) & =(\nabla v, \nabla v)_{\Sigma}+(\kappa v, v)_{\Gamma} \geq \min \left(1, \frac{1}{2} \kappa_{\min }\right) C_{P F}^{-1}(\Sigma)\|v\|_{L^{2}(\Sigma)}^{2}+\frac{1}{2} \kappa_{m i n}\|v\|_{L^{2}(\Gamma)}^{2} \\
& \geq \frac{1}{2} \min \left(2 C_{P F}^{-1}(\Sigma), C_{P F}^{-1}(\Sigma) \kappa_{\min }, \kappa_{\min }\right)\|v\|_{\mathrm{o}}^{2}, \forall v \in H^{1}(\Sigma)
\end{aligned}
$$

As a result of that we obtain,

$$
\begin{aligned}
\frac{1}{2} \min \left(2 C_{P F}^{-1}(\Sigma), C_{P F}^{-1}(\Sigma) \kappa_{\text {min }}, \kappa_{\text {min }}\right)\left\|z_{\text {ref }}^{(1)}\right\|_{\circ}^{2} & \leq a_{\text {ref }}\left(z_{\text {ref }}^{(1)}, z_{\text {ref }}^{(1)}\right)=\left(e, z_{\text {ref }}^{(1)}\right)_{\Sigma} \\
& \leq\|e\|_{L^{2}(\Sigma)}\left\|z_{\text {ref }}^{(1)}\right\|_{L^{2}(\Sigma)} \leq\|e\|_{L^{2}(\Sigma)}\left\|z_{\text {ref }}^{(1)}\right\|_{\circ}
\end{aligned}
$$

from which it follows that

$$
\left\|a_{\mathrm{ref}}^{(1)}\right\|_{\circ}=\frac{2}{\min \left(2 C_{P F}^{-1}(\Sigma), C_{P F}^{-1}(\Sigma) \kappa_{\min }, \kappa_{\min }\right)}
$$

and for Lemma 4.4 the constant $\left\|a_{\text {ref }}^{(1)}\right\|_{\circ}$ is bounded for $\epsilon \rightarrow 0$.

\subsection{Analysis of the modeling error relative to the domain (assumption A2)}

This component of the modeling error arises because we identify the domain $\Omega_{\oplus}$ of (1) with the entire domain $\Omega$. Let us assume for simplicity that $f \in H^{1}\left(\Omega_{\oplus}\right)$ and let us denote by $\mathcal{E}_{\Sigma} f$ its extension to $H^{1}(\Sigma)$. In this case the reference and reduced models are respectively

$$
\begin{array}{r}
\text { find } u_{\oplus} \in H_{\partial \Omega}^{1}\left(\Omega_{\oplus}\right):\left(\nabla u_{\oplus}, \nabla v\right)_{\Omega_{\oplus}}+\left(\kappa u_{\oplus}, v\right)_{\Gamma}=(f, v)_{\Omega_{\oplus}}+(\kappa U, v)_{\Gamma}, \forall v \in H_{\partial \Omega}^{1}\left(\Omega_{\oplus}\right), \\
\text { find } u^{(2)} \in H_{0}^{1}(\Omega):\left(\nabla u^{(2)}, \nabla v\right)_{\Omega}+\left(\kappa u^{(2)}, v\right)_{\Gamma}=\left(\left(\mathcal{I}_{\Omega_{\oplus}}+\mathcal{E}_{\Sigma}\right) f, v\right)_{\Omega}+(\kappa U, v)_{\Gamma}, \forall v \in H_{0}^{1}(\Omega) .
\end{array}
$$

We extend the solution of (45) from $\Omega_{\oplus}$ to $\Omega$, with the extension operator $\mathcal{E}_{\Sigma}$ which takes the function $u_{\oplus}$ on $\Omega_{\oplus}$ and extends it to the interior of the domain. Then, the reference solution relative to assumption A2 is $u_{\text {ref }}^{(2)}=\left(\mathcal{I}_{\Omega_{\oplus}}+\mathcal{E}_{\Sigma}\right) u_{\oplus} \in H_{0}^{1}(\Omega)$. The solution of the reduced problem is instead $u^{(2)} \in H_{0}^{1}(\Omega)$. The functional space where we set the modeling error is $X^{(2)}=H_{0}^{1}(\Omega)$ and error is $e^{(2)}=u_{\mathrm{ref}}^{(2)}-u^{(2)}=\left(\mathcal{I}_{\Omega_{\oplus}}+\mathcal{E}_{\Sigma}\right) u_{\oplus}-u^{(2)} \in$ $H_{0}^{1}(\Omega)$. Then, subtracting (46) from (45), it is straightforward to determine the expression of the difference operators $d^{(2)}(u, v)$ and $l^{(2)}(v)$,

$$
d^{(2)}(u, v)=-(\nabla u, \nabla v)_{\Sigma}, \quad l^{(2)}(v)=-\left(\mathcal{E}_{\Sigma} f, v\right)_{\Sigma} \quad \forall u, v \in H_{0}^{1}(\Omega) .
$$

Lemma 4.8. The operators $d^{(2)}(\cdot, \cdot)$ and $l^{(2)}(\cdot)$ satisfy Property 4.1 with the norms $\|\cdot\|_{\star}=\|\cdot\|_{H^{\frac{3}{2}-\eta}(\Omega)}$, fora any $\eta>0$, and $\|\cdot\|_{\circ}=\|\cdot\|_{H^{1}\left(\Omega_{\oplus}\right)}$ and inequality (36) is satisfied with constants

$$
\left\|d^{(2)}\right\|_{\star \circ}=C_{E}\left(S C_{\mathcal{D}}\right)^{\frac{1-2 \eta}{6}} \epsilon^{\frac{1-2 \eta}{3}} C(1 / 2-\eta, 3 /(1+\eta), \Omega), \quad\left\|l^{(2)}\right\|_{\circ}=\left(1+C_{E}\right) C_{E}\left(S C_{\mathcal{D}}\right)^{\frac{1}{2}} \epsilon\|f\|_{H^{1}\left(\Omega_{\oplus}\right)} .
$$

Proof. Let us start with the upper bound for $d^{(2)}(u, v)$, that is $d^{(2)}(u, v) \leq\|\nabla u\|_{L^{2}(\Sigma)}\|\nabla v\|_{L^{2}(\Sigma)}$. Let us assume that $u \in H^{k}(\Omega)$ with $1<k \leq 2$, then $\nabla u \in H^{k-1}(\Omega)$ and for Sobolev embedding theorem we have $\nabla u \in L^{p^{*}}(\Omega)$ with $p^{*}=6 /(5-2 k)$. Then we apply Hölder inequality and (13) as follows,

$$
\begin{aligned}
\|\nabla u\|_{L^{2}(\Sigma)} & =\left(\int_{\Sigma}(\nabla u)^{2} d \omega\right)^{\frac{1}{2}} \leq\left(\int_{\Sigma} 1 d \omega\right)^{\frac{1}{2 q}}\left(\int_{\Sigma}(\nabla u)^{2 r} d \omega\right)^{\frac{1}{2 r}} \\
& \leq|\Sigma|^{\frac{1}{2 q}}\|\nabla u\|_{L^{2 r}(\Sigma)} \leq\left(S C_{\mathcal{D}}\right)^{\frac{1}{2 q}} \epsilon^{\frac{1}{q}}\|\nabla u\|_{L^{2 r}(\Sigma)}
\end{aligned}
$$

where $q, r$ must satisfy $1 / q+1 / r=1$. The maximum exponent for which the previous inequality holds true is $p^{*}=2 r=6 /(5-2 k)$. Then, $r=3 /(5-2 k)$ and $1 / q=2(k-1) / 3$. Denoting with $C\left(k-1, p^{*}, \Omega\right)$ the 
constant of the Sobolev inequality of the embedding $H^{k-1}(\Omega) \subset L^{p^{*}}(\Omega)$, we have

$$
\begin{aligned}
\|\nabla u\|_{L^{2}(\Sigma)} & \leq\left(S C_{\mathcal{D}}\right)^{\frac{1}{2 q}} \epsilon^{\frac{1}{q}}\|\nabla u\|_{L^{p^{*}}(\Sigma)} \leq\left(S C_{\mathcal{D}}\right)^{\frac{1}{2 q}} \epsilon^{\frac{1}{q}}\|\nabla u\|_{L^{p^{*}}(\Omega)} \\
& \leq\left(S C_{\mathcal{D}}\right)^{\frac{1}{2 q}} \epsilon^{\frac{1}{q}} C\left(k-1, p^{*}, \Omega\right)\|\nabla u\|_{H^{k-1}(\Omega)} \leq\left(S C_{\mathcal{D}}\right)^{\frac{1}{2 q}} \epsilon^{\frac{1}{q}} C\left(k-1, p^{*}, \Omega\right)\|u\|_{H^{k}(\Omega)} .
\end{aligned}
$$

Being $u^{(2)} \in H^{\frac{3}{2}-\eta}(\Omega)$ and $z_{\text {ref }}^{(2)} \in H_{\partial \Omega}^{1}\left(\Omega_{\oplus}\right)$, we obtain,

$$
d\left(u^{(2)},\left(\mathcal{I}_{\Omega_{\oplus}}+\mathcal{E}_{\Sigma}\right) z_{\mathrm{ref}}\right) \leq C_{E}\left(S C_{\mathcal{D}}\right)^{\frac{1-2 \eta}{6}} \epsilon^{\frac{1-2 \eta}{3}} C(1 / 2-\eta, 3 /(1+\eta), \Omega)\left\|u^{(2)}\right\|_{H^{\frac{3}{2}-\eta}(\Omega)}\left\|z_{\mathrm{ref}}\right\|_{H^{1}\left(\Omega_{\oplus}\right)} .
$$

Proceeding similarly, using the minimal regularity requirement $\mathcal{E}_{\Sigma} f \in H^{1}(\Sigma)$ combined with Hölder inequality and Sobolev embeddings, namely $H^{1}(\Sigma) \subset L^{6}(\Sigma)$, we have

$$
\begin{aligned}
l\left(\left(\mathcal{I}_{\Omega_{\oplus}}+\mathcal{E}_{\Sigma}\right) z_{\mathrm{ref}}\right) & =-\left(\mathcal{E}_{\Sigma} f,\left(\mathcal{I}_{\Omega_{\oplus}}+\mathcal{E}_{\Sigma}\right) z_{\mathrm{ref}}\right)_{\Sigma} \leq C_{E}\left\|\mathcal{E}_{\Sigma} f\right\|_{L^{2}(\Sigma)}\left\|z_{\mathrm{ref}}\right\|_{H^{1}\left(\Omega_{\oplus}\right)} \\
& \leq C_{E}\left(S C_{\mathcal{D}}\right)^{\frac{1}{3}} \epsilon^{\frac{2}{3}}\left\|\mathcal{E}_{\Sigma} f\right\|_{L^{6}(\Sigma)}\left\|z_{\mathrm{ref}}\right\|_{H^{1}\left(\Omega_{\oplus}\right)} \leq C_{E}\left(S C_{\mathcal{D}}\right)^{\frac{1}{3}} \epsilon^{\frac{2}{3}}\left\|\left(\mathcal{I}_{\Omega_{\oplus}}+\mathcal{E}_{\Sigma}\right) f\right\|_{L^{6}(\Omega)}\left\|z_{\mathrm{ref}}\right\|_{H^{1}\left(\Omega_{\oplus}\right)} \\
& \leq C_{E}\left(1+C_{E}\right)\left(S C_{\mathcal{D}}\right)^{\frac{1}{3}} \epsilon^{\frac{2}{3}} C(1,6, \Omega)\|f\|_{H^{1}\left(\Omega_{\oplus}\right)}\left\|z_{\mathrm{ref}}\right\|_{H^{1}\left(\Omega_{\oplus}\right)},
\end{aligned}
$$

being $C(1,6, \Omega)$ the constant in the Sobolev embedding of $H^{1}(\Omega)$ in $L^{6}(\Omega)$.

Lemma 4.9. The solution of the reduced problem $u^{(2)}$ satisfies the Property 4.2 with the norm $\|\cdot\|_{\star}=$ $\|\cdot\|_{H^{\frac{3}{2}-\eta}(\Omega)}$, for any $\eta>0$ and the inequality (37) is satisfied with the positive constant, uniformly upper bounded for $\epsilon$,

$$
\left\|a^{(2)}\right\|_{\star}=2 C_{R} \max \left[\left(1+C_{E}\right),\left(1+C_{P}(\Omega)\right)\left(1+C_{E}\right) \sqrt{C_{T}(\Gamma, \Omega)},\|\kappa\|_{L^{\infty}} \sqrt{C_{T}(\Gamma, \Omega)},\|\kappa\|_{L^{\infty}} \sqrt{C^{\prime} T_{T}\left(\Gamma, \Omega_{\oplus}\right)}\right] .
$$

Proof. We notice that (46) in the strong form reads as

$$
-\Delta u^{(2)}=\left(\mathcal{I}_{\Omega_{\oplus}}+\mathcal{E}_{\Sigma}\right) f-\kappa\left(u^{(2)}-U\right) \delta_{\Gamma} \text { in } \Omega, \quad u^{(2)}=0 \text { on } \partial \Omega .
$$

Then, owing to Theorem 3.11, $u^{(2)} \in H^{\frac{3}{2}-\eta}(\Omega) \cap H_{0}^{1}(\Omega)$ and using (30) we obtain the following upper bound,

$$
\left\|u^{(2)}\right\|_{H^{\frac{3}{2}-\eta}(\Omega)} \leq C_{R}\left\|\left(\mathcal{I}_{\Omega_{\oplus}}+\mathcal{E}_{\Sigma}\right) f-\kappa\left(u^{(2)}-U\right) \delta_{\Gamma}\right\|_{H^{-\frac{1}{2}-\eta}(\Omega)} .
$$

By the Sobolev embedding $H^{1}(\Omega) \subset H^{-\frac{1}{2}-\eta}(\Omega)$ we have,

$$
\left\|\left(\mathcal{I}_{\Omega_{\oplus}}+\mathcal{E}_{\Sigma}\right) f\right\|_{H^{-\frac{1}{2}-\eta}(\Omega)} \leq\left(1+C_{E}\right)\|f\|_{H^{1}\left(\Omega_{\oplus}\right)} .
$$

From the definition of $\|\cdot\|_{H^{-\frac{1}{2}-\eta}(\Omega)}$, we have

$$
\left\|\kappa U \delta_{\Gamma}\right\|_{H^{-\frac{1}{2}-\eta}(\Omega)}=\sup _{\substack{w \in H^{\frac{1}{2}+\eta}(\Omega) \\ w \neq 0}} \frac{\left(\kappa U \delta_{\Gamma}, w\right)_{\Omega}}{\|w\|_{H^{\frac{1}{2}+\eta}(\Omega)}} .
$$

Then the following upper bound holds,

$$
\left(\kappa U \delta_{\Gamma}, w\right)_{\Omega}=(\kappa U, w)_{\Gamma} \leq\|\kappa\|_{L^{\infty}}\|U\|_{L^{2}(\Gamma)}\|w\|_{L^{2}(\Gamma)} \leq\|\kappa\|_{L^{\infty}} \sqrt{C_{T}^{\prime}(\Gamma, \Omega)}\|U\|_{L^{2}(\Lambda),|\partial \mathcal{D}|}\|w\|_{H^{\frac{1}{2}+\eta}(\Omega)},
$$

where $C_{T}^{\prime}(\Gamma, \Omega)$ is the constant in the trace inequality from $L^{2}(\Gamma)$ to $H^{\frac{1}{2}+\eta}(\Omega)$. Proceeding similarly, we obtain the following estimate,

$$
\left\|\kappa u^{(2)} \delta_{\Gamma}\right\|_{H^{-\frac{1}{2}-\eta}(\Omega)} \leq\|\kappa\|_{L^{\infty}(\Gamma)} \sqrt{C_{T}^{\prime}(\Gamma, \Omega)}\left\|u^{(2)}\right\|_{L^{2}(\Gamma)} \leq\|\kappa\|_{L^{\infty}(\Gamma)} \sqrt{C_{T}(\Gamma, \Omega) C_{T}^{\prime}(\Gamma, \Omega)}\left\|u^{(2)}\right\|_{H^{1}(\Omega)} .
$$


Then, we notice that problem (46) satisfies the following stability property in $H^{1}(\Omega)$,

$$
\begin{gathered}
\left(1+C_{P}(\Omega)\right)^{-1}\left\|u^{(2)}\right\|_{H^{1}(\Omega)}^{2} \leq\left(\nabla u^{(2)}, \nabla u^{(2)}\right)_{\Omega}+\left(\kappa u^{(2)}, u^{(2)}\right)_{\Gamma} \leq\left(\left(\mathcal{I}_{\Omega_{\oplus}}+\mathcal{E}_{\Sigma}\right) f, u^{(2)}\right)_{\Omega}+\left(\kappa U, u^{(2)}\right)_{\Gamma} \\
\leq\left(1+C_{E}\right)\|f\|_{H^{1}\left(\Omega_{\oplus}\right)}\left\|u^{(2)}\right\|_{H^{1}(\Omega)}+\|\kappa\|_{L^{\infty}(\Gamma)} \sqrt{C_{T}(\Gamma, \Omega)}\|U\|_{L^{2}(\Lambda),|\partial \mathcal{D}|}\left\|u^{(2)}\right\|_{H^{1}(\Omega)},
\end{gathered}
$$

that is,

$$
\left\|u^{(2)}\right\|_{H^{1}(\Omega)} \leq\left(1+C_{P}(\Omega)\right) \max \left(\left(1+C_{E}\right),\|\kappa\|_{L^{\infty}(\Gamma)} \sqrt{C_{T}(\Gamma, \Omega)}\right)\left(\|f\|_{H^{1}\left(\Omega_{\oplus}\right)}+\|U\|_{L^{2}(\Lambda),|\partial \mathcal{D}|}\right) .
$$

Combining the upper bounds for each term on the right hand side of (48) we obtain the desired result,

$$
\begin{array}{r}
\left\|u^{(2)}\right\|_{\star} \leq 2 C_{R} \max \left(\left(1+C_{E}\right),\|\kappa\|_{L^{\infty}} \sqrt{C_{T}^{\prime}\left(\Gamma, \Omega_{\oplus}\right)},\|\kappa\|_{L^{\infty}} \sqrt{C_{T}(\Gamma, \Omega) C_{T}^{\prime}\left(\Gamma, \Omega_{\oplus}\right)}\left(1+C_{P}\right)\left(1+C_{E}\right),\right. \\
\left.\|\kappa\|_{L^{\infty}}^{2} C_{T}(\Gamma, \Omega) \sqrt{C_{T}^{\prime}\left(\Gamma, \Omega_{\oplus}\right)}\right)\left(\|f\|_{H^{1}\left(\Omega_{\oplus}\right)}+\|U\|_{L^{2}(\Lambda),|\partial \mathcal{D}|}\right) .
\end{array}
$$

Lemma 4.10. The solution of the reference dual problem, that is to find $z_{\mathrm{ref}}^{(2)} \in H_{\partial \Omega}^{1}\left(\Omega_{\oplus}\right)$ such that

$$
\left(\nabla v, \nabla z_{\mathrm{ref}}^{(2)}\right)_{\Omega_{\oplus}}+\left(\kappa v, z_{\mathrm{ref}}^{(2)}\right)_{\Gamma}=j^{(2)}(v), \forall v \in H_{\partial \Omega}^{1}\left(\Omega_{\oplus}\right),
$$

with $j^{(2)}(v)=\left(e^{(2)},\left(\mathcal{I}_{\Omega_{\oplus}}+\mathcal{E}_{\Sigma}\right) v\right)_{\Omega}$, satisfies the following inequality,

$$
\begin{aligned}
\left(1+C_{P}\left(\Omega_{\oplus}\right)\right)^{-1}\left\|z_{\text {ref }}^{(2)}\right\|_{\Omega_{\oplus}}^{2} & \leq\left(\nabla z_{\text {ref }}^{(2)}, \nabla z_{\text {ref }}^{(2)}\right)_{\Omega_{\oplus}}+\left(\kappa z_{\text {ref }}^{(2)}, z_{\text {ref }}^{(2)}\right)_{\Gamma}=\left(e^{(2)},\left(\mathcal{I}_{\Omega_{\oplus}}+\mathcal{E}_{\Sigma}\right) z_{\text {ref }}^{(2)}\right)_{\Omega} \\
& \leq\left(1+C_{E}\right)\left\|e^{(2)}\right\|_{L^{2}(\Omega)}\left\|z_{\text {ref }}^{(2)}\right\|_{H^{1}\left(\Omega_{\oplus}\right)} .
\end{aligned}
$$

As a result, Property 4.2 is satisfied with the norm $\|\cdot\|_{\circ}=\|\cdot\|_{H^{1}\left(\Omega_{\oplus}\right)}$ and with the constant,

$$
\left\|a_{\mathrm{ref}}^{(2)}\right\|_{\circ}=\left(1+C_{P}\left(\Omega_{\oplus}\right)\right)\left(1+C_{E}\right) .
$$

\subsection{Analysis of the modeling error of the transmission conditions (assumption A3)}

The interface conditions between $\Sigma$ and $\Omega_{\oplus}$ must be adapted to the topological model reduction of the problem on $\Sigma$. This is achieved by averaging the solution $u_{\oplus}$ on cross sections of the interface $\partial \mathcal{D}(s)$ before enforcing the interface condition between $u_{\oplus}$ and $U$. The error that arises in this process corresponds to neglect the fluctuations of $u_{\oplus}$ at the interface, as stated in assumption A3. In this case, the reference problem is (46), with solution $u_{\mathrm{ref}}^{(3)}=u^{(2)}$, while the reduced problem is (8) so that $u^{(3)}=u$, being $u$ the solution of the final reduced model on $\Omega$. For the sake of clarity, as was done in the previous cases, we report the reference and the reduced problems here:

$$
\begin{aligned}
\text { find } u^{(2)} \in H_{0}^{1}(\Omega): & \left(\nabla u^{(2)}, \nabla v\right)_{\Omega}+\left(\kappa u^{(2)}, v\right)_{\Gamma}=\left(\left(\mathcal{I}_{\Omega_{\oplus}}+\mathcal{E}_{\Sigma}\right) f, v\right)_{\Omega}+(\kappa U, v)_{\Gamma}, \forall v \in H_{0}^{1}(\Omega), \\
\text { find } u \in H_{0}^{1}(\Omega): & (\nabla u, \nabla v)_{\Omega}+(\kappa \bar{u}, \bar{v})_{\Lambda,|\partial \mathcal{D}|}=\left(\left(\mathcal{I}_{\Omega_{\oplus}}+\mathcal{E}_{\Sigma}\right) f, v\right)_{\Omega}+(\kappa U, \bar{v})_{\Lambda,|\partial \mathcal{D}|}, \forall v \in H_{0}^{1}(\Omega) .
\end{aligned}
$$

The modeling error is easily defined as $e^{(3)}=u_{\mathrm{ref}}^{(3)}-u^{(3)}=u^{(2)}-u \in H_{0}^{1}(\Omega)$ for any $u, v \in H_{0}^{1}(\Omega)$. Provided that $U$ is uniformly extended from $H^{1}(\Lambda)$ to $H^{1}(\Sigma)$ the difference operator between (51) and (52) is

$$
\begin{aligned}
d^{(3)}(u, v) & =(\kappa u, v)_{\Gamma}-(\kappa \bar{u}, \bar{v})_{\Lambda,|\partial \mathcal{D}|}=(\kappa(\mathcal{I}-\overline{(\cdot)}) u, v)_{\Gamma} \\
l^{(3)}(v) & =(\kappa U, v)_{\Gamma}-(\kappa U, \bar{v})_{\Lambda,|\partial \mathcal{D}|}=0 .
\end{aligned}
$$


Lemma 4.11. Under the assumption that $u \in H_{0}^{1}(\Omega) \cap H^{\frac{3}{2}}(\Sigma)$, the operators $d^{(3)}(\cdot, \cdot)$ and $l^{(3)}(\cdot)$ satisfy Property 4.1 with the norms $\|\cdot\|_{\star}=\|\cdot\|_{\circ}=\|\cdot\|_{H^{1}(\Omega)}$ and with constants

$$
\left\|d^{(3)}\right\|_{\star \circ}=\|\kappa\|_{L^{\infty}} \sqrt{C_{T}(\Gamma, \Omega)} \sqrt{C_{S} \epsilon}, \quad\left\|l^{(3)}\right\|_{\circ}=0 .
$$

In particular, if we take into account the dependence of $C_{T}(\Gamma, \Omega)$ on $\epsilon$, using Lemma 3.4, we obtain that $\left\|d^{(3)}\right\|_{\star \circ}=\mathcal{O}\left(\epsilon^{1-\frac{1}{2(2-\delta)}}\right)$.

Proof. Since $u \in H^{\frac{3}{2}}(\Sigma)$, we have that $\left.u\right|_{\mathcal{D}(s)} \in H^{1}(\mathcal{D}(s))$ for any $s \in(0, S)$. Then we can apply Stekloff inequality on any cross section of $\Sigma$. Combining it it with Cauchy-Schwarz and trace inequalities, we obtain,

$$
\begin{aligned}
d^{(3)}\left(u, z_{\mathrm{ref}}\right) & =\left(\kappa(\mathcal{I}-\overline{(\cdot)}) u, z_{\mathrm{ref}}\right)_{\Gamma} \leq\|\kappa\|_{L^{\infty}} \sqrt{C_{S} \epsilon}\left(\int_{\Lambda} \int_{\mathcal{D}(s)}\left(\nabla_{\mathcal{D}} u\right)^{2} d \sigma d s\right)^{\frac{1}{2}}\left\|z_{\mathrm{ref}}\right\|_{L^{2}(\Gamma)} \\
& \leq\|\kappa\|_{L^{\infty}} \sqrt{C_{S} \epsilon}\|\nabla u\|_{L^{2}(\Sigma)}\left\|z_{\mathrm{ref}}\right\|_{L^{2}(\Gamma)} \leq\|\kappa\|_{L^{\infty}} \sqrt{C_{T}(\Gamma, \Omega)} \sqrt{C_{S} \epsilon}\|u\|_{H^{1}(\Omega)}\left\|z_{\mathrm{ref}}\right\|_{H^{1}(\Omega)} .
\end{aligned}
$$

Lemma 4.12. The solution $u^{(3)}$ of problem (52) satisfies the following inequality

$$
\left\|u^{(3)}\right\|_{H^{1}(\Omega)} \leq\left(1+C_{P}(\Omega)\right) \max \left(\left(1+C_{E}\right),\|\kappa\|_{L^{\infty}} \sqrt{C_{T}(\Gamma, \Omega)}\right)\left(\|f\|_{H^{1}\left(\Omega_{\oplus}\right)}+\|U\|_{L^{2}(\Lambda),|\partial \mathcal{D}|}\right) .
$$

As result, Property 4.2 is satisfied with the norm $\|\cdot\|_{\star}=\|\cdot\|_{H^{1}(\Omega)}$ and with the constant

$$
\left\|a^{(3)}\right\|_{\star}=\left(1+C_{P}(\Omega)\right) \max \left(\left(1+C_{E}\right),\|\kappa\|_{L^{\infty}} \sqrt{C_{T}(\Gamma, \Omega)}\right) .
$$

Proof. From problem (52) we obtain,

$$
\begin{aligned}
\left(1+C_{P}(\Omega)\right)^{-1}\left\|u^{(3)}\right\|_{H^{1}(\Omega)}^{2} & \leq(\nabla u, \nabla u)_{\Omega}+(\kappa \bar{u}, \bar{u})_{\Lambda,|\partial \mathcal{D}|} \\
& \leq\left\|\left(\mathcal{I}_{\Omega_{\oplus}}+\mathcal{E}_{\Sigma}\right) f\right\|_{L^{2}(\Omega)}\left\|u^{(3)}\right\|_{L^{2}(\Omega)}+\|\kappa\|_{L^{\infty}}\|U\|_{L^{2}(\Lambda),|\partial \mathcal{D}|}\|\bar{u}\|_{L^{2}(\Lambda),|\partial \mathcal{D}|} \\
& \leq\left(1+C_{E}\right)\|f\|_{L^{2}\left(\Omega_{\oplus}\right)}\left\|u^{(3)}\right\|_{H^{1}(\Omega)}+\|\kappa\|_{L^{\infty}} \sqrt{C_{T}(\Gamma, \Omega)}\|U\|_{L^{2}(\Lambda),|\partial \mathcal{D}|}\left\|u^{(3)}\right\|_{H^{1}(\Omega)},
\end{aligned}
$$

from which it follows that

$$
\left\|u^{(3)}\right\|_{H^{1}(\Omega)} \leq\left(1+C_{P}(\Omega)\right) \max \left(1+C_{E},\|\kappa\|_{L^{\infty}} \sqrt{C_{T}(\Gamma, \Omega)}\right)\left(\|f\|_{H^{1}\left(\Omega_{\oplus}\right)}+\|U\|_{L^{2}(\Lambda),|\partial \mathcal{D}|}\right) .
$$

Consequently,

$$
\left\|a^{(3)}\right\|_{\star}=\left(1+C_{P}(\Omega)\right) \max \left(1+C_{E},\|\kappa\|_{L^{\infty}} \sqrt{C_{T}(\Gamma, \Omega)}\right) .
$$

Lemma 4.13. The solution of the reference dual problem, that is to find $z_{\mathrm{ref}}^{(3)} \in H_{0}^{1}(\Omega)$ such that

$$
\left(\nabla v, \nabla z_{\text {ref }}^{(3)}\right)_{\Omega}+\left(\kappa v, z_{\text {ref }}^{(3)}\right)_{\Gamma}=j^{(3)}(v), \forall v \in H_{0}^{1}(\Omega),
$$

with $j^{(3)}(v)=\left(e^{(3)}, v\right)_{\Omega}$, satisfies the following inequality

$$
\left\|z_{\text {ref }}^{(3)}\right\|_{H^{1}(\Omega)} \leq\left(1+C_{P}(\Omega)\right)\left\|e^{(3)}\right\|_{L^{2}(\Omega)} .
$$

As result, Property 4.2 is satisfied with the norm $\|\cdot\|_{\circ}=\|\cdot\|_{H^{1}(\Omega)}$ and the constant

$$
\left\|a_{\text {ref }}^{(3)}\right\|_{\circ}=1+C_{P}(\Omega) .
$$




\subsection{Conclusions of the model error analysis}

In the previous sections we have analyzed three different components of the model error. In particular, we recall that

$$
\begin{aligned}
& e^{(1)}=u_{\mathrm{ref}}^{(1)}-u^{(1)}=u_{\ominus}-U \in H^{1}(\Sigma) \\
& e^{(2)}=u_{\mathrm{ref}}^{(2)}-u^{(2)}=\left(\mathcal{I}_{\Omega_{\oplus}}+\mathcal{E}_{\Sigma}\right) u_{\oplus}-u^{(2)} \in H_{0}^{1}(\Omega) \\
& e^{(3)}=u_{\mathrm{ref}}^{(3)}-u^{(3)}=u^{(2)}-u \in H_{0}^{1}(\Omega)
\end{aligned}
$$

As a result, the total model error, on $\Sigma$ and $\Omega$ respectively can be straightforwardly decomposed as

$$
e_{\Sigma}+e_{\Omega}=\left(u_{\ominus}-U\right)+\left(\left(\mathcal{I}_{\Omega_{\oplus}}+\mathcal{E}_{\Sigma}\right) u_{\oplus}-u\right)=e^{(1)}+e^{(2)}+e^{(3)},
$$

with $e_{\Sigma}=e^{(1)} \in H^{1}(\Sigma)$ and $e_{\Omega}=e^{(2)}+e^{(3)} \in H_{0}^{1}(\Omega)$. Then, for a suitable choice of the model error output functionals used in the reference dual problems, if Properties (4.1) and (4.2) are satisfied, the following inequality holds true,

$$
j^{(k)}(e)=\left\|e^{(k)}\right\|_{L^{2}} \leq\left(\left\|l^{(k)}\right\|_{\circ}+\left\|d^{(k)}\right\|_{\star \circ}\left\|a^{(k)}\right\|_{\star}\|\mathcal{F}\|_{X^{\prime}}\right)\left\|a_{\text {ref }}^{(k)}\right\|_{\circ}, \quad k=1,2,3 .
$$

For $e_{\Sigma}$, if $u_{\oplus} \in H_{\partial \Omega}^{1}\left(\Omega_{\oplus}\right) \cap H^{2}\left(\Omega_{\oplus}\right)$, we have

$$
\left\|e^{(1)}\right\|_{L^{2}(\Sigma)} \leq\left(\left\|l^{(1)}\right\|_{\circ}+\left\|d^{(1)}\right\|_{\star \circ}\left\|a^{(1)}\right\|_{\star}\|\mathcal{F}\|_{X^{\prime}}\right)\left\|a_{\text {ref }}^{(1)}\right\|_{\circ}=\left\|l^{(1)}\right\|_{\circ}\left\|a_{\text {ref }}^{(1)}\right\|_{\circ}=\mathcal{O}\left(\epsilon^{\frac{1}{2}}\right),
$$

because $\left\|l^{(1)}\right\|_{\circ}=\mathcal{O}\left(\epsilon^{\frac{1}{2}}\right)$ for Lemma $4.6,\left\|d^{(1)}\right\|_{\star \circ}=0$ for Lemma 4.5 and $\left\|a_{\text {ref }}^{(1)}\right\|_{\circ}$ is uniformly bounded with respect to $\epsilon$. For $e_{\Omega}$, if $u \in H_{0}^{1}(\Omega) \cap H^{\frac{3}{2}}(\Sigma)$, from Lemmas 4.8, 4.9, 4.10 we obtain

$$
\left\|e^{(2)}\right\|_{L^{2}(\Omega)} \leq\left(\left\|l^{(2)}\right\|_{\circ}+\left\|d^{(2)}\right\|_{\star \circ}\left\|a^{(2)}\right\|_{\star}\left(\|f\|_{H^{1}\left(\Omega_{\oplus}\right)}+\|U\|_{L^{2}(\Lambda),|\partial \mathcal{D}|}\right)\right)\left\|a_{\mathrm{ref}}^{(2)}\right\|_{\circ}=\mathcal{O}\left(\epsilon^{\frac{1-2 \eta}{3}}\right),
$$

and from Lemmas 4.11, 4.12, 4.13 we have

$$
\left\|e^{(3)}\right\|_{L^{2}(\Omega)} \leq\left\|d^{(3)}\right\|_{\star 0}\left\|a^{(3)}\right\|_{\star}\left(\|f\|_{H^{1}\left(\Omega_{\oplus}\right)}+\|U\|_{L^{2}(\Lambda),|\partial \mathcal{D}|}\right)\left\|a_{\text {ref }}^{(3)}\right\|_{\circ}=\mathcal{O}\left(\epsilon^{\frac{1}{2}}\right) .
$$

By combining the previous upper bounds for $\left\|e^{(2)}\right\|_{L^{2}(\Omega)}$ and $\left\|e^{(3)}\right\|_{L^{2}(\Omega)}$ we obtain the following estimate for $\left\|e_{\Omega}\right\|_{L^{2}(\Omega)}$

$$
\left\|e_{\Omega}\right\|_{L^{2}} \leq\left\|l^{(2)}\right\|_{\circ}\left\|a_{\mathrm{ref}}^{(2)}\right\|_{\circ}+\left(\left\|d^{(2)}\right\|_{\star \circ}\left\|a^{(2)}\right\|_{\star}\left\|a_{\mathrm{ref}}^{(2)}\right\|_{\circ}+\left\|d^{(3)}\right\|_{\star \circ}\left\|a^{(3)}\right\|_{\star}\left\|a_{\mathrm{ref}}^{(3)}\right\|_{\circ}\right)\left(\|f\|_{H^{1}\left(\Omega_{\oplus}\right)}+\|U\|_{L^{2}(\Lambda),|\partial \mathcal{D}|}\right)
$$

which entails that $\left\|e_{\Omega}\right\|_{L^{2}(\Omega)}=\mathcal{O}\left(\epsilon^{\frac{1-2 \eta}{3}}\right)$ as $\epsilon \rightarrow 0$.

Remark 4.14. The model error analysis relies on two additional regularity assumptions, $u_{\oplus} \in H^{2}\left(\Omega_{\oplus}\right)$ and $u \in H^{\frac{3}{2}}(\Sigma)$. These are technical assumptions required to use the Stekloff inequality (39). The former one is more restrictive because $\mathcal{E}_{\Sigma} u_{\oplus} \in H^{\frac{3}{2}}\left(\Omega_{\oplus}\right)$ requires $u_{\oplus} \in H^{2}\left(\Omega_{\oplus}\right)$ since Lemma 4.3 works for Sobolev spaces with integer index. We remark that none of the constants appearing in (56), (57) and (58) involve neither $\left\|u_{\oplus}\right\|_{H^{2}\left(\Omega_{\oplus}\right)}$ nor $\|u\|_{H^{3 / 2}(\Sigma)}$.

Furthermore, we observe that these regularity assumptions are local regularity properties on subregions of $\Omega$ that do not cross the surface $\Gamma$. As a consequence, these properties can be justified with classical regularity results for elliptic equations with regular coefficients and on domains with smooth boundary, see for example Theorem 3.10 of [15] and references therein. We report below a sketch of the proofs, although we do not claim to be fully rigorous.

For the former assumption, we consider problem (1) and we fix $u_{\ominus} \in H^{1}(\Sigma)$. Then $u_{\oplus}$ solves a Poisson problem with Robin boundary conditions on the inner boundary of $\Omega_{\oplus}$ and homogeneous Dirichlet conditions 
on the outer boundary, which are disjoint. Given a $\mathcal{C}^{2}$ regular domain, a right hand side $f \in L^{2}\left(\Omega_{\oplus}\right)$ and a forcing term of the Robin condition that is $g=\kappa u_{\ominus} \in H^{\frac{1}{2}}(\Gamma)$, we conclude that $u_{\oplus} \in H^{2}\left(\Omega_{\oplus}\right)$.

For the latter assumption, we observe from equation (8) that the restriction of $u$ to $\Sigma$ solves the following problem in the weak sense,

$$
\begin{cases}-\Delta u=f & \text { in } \Sigma, \\ -\nabla u \cdot \boldsymbol{n}=\kappa \mathcal{E}_{\Gamma}(\bar{u}-U) & \text { on } \Gamma, \\ -\nabla u \cdot \boldsymbol{n}=0 & \text { on } \partial \Sigma \backslash \Gamma .\end{cases}
$$

Given the standard $H^{1}$-regularity of Theorem 3.3 we have $\mathcal{E}_{\Gamma}(\bar{u}-U) \in H^{\frac{1}{2}}(\Gamma)$. If this function smoothly vanishes approaching $\partial \Sigma \backslash \Gamma$, then this problem is a Neumann problem with $H^{\frac{1}{2}}$-regular data on the boundary. As a result, standard $H^{2}$-regularity also apply to the restriction of $u$ on $\Sigma$.

\section{NUMERICAL EXPERIMENTS}

The purpose of this section is twofold. First we introduce the basic aspects of the numerical approximation of the problem by means of finite elements. In this context, we validate the numerical solver on a simple problem for which the solution is available explicitly. Second, we use the numerical method to calculate a local error estimator of the modeling error. This is a preliminary attempt of a posteriori model error analysis, that is used here for a qualitative investigation of the spatial distribution of the model error.

\subsection{Finite element approximation}

Let us consider a quasi-uniform partition $\mathcal{T}_{\Omega}^{h}$ of $\Omega$ and an admissible partition $\mathcal{T}_{\Lambda}^{h}$ of $\Lambda$ with comparable characteristic size, denoted by $h$, and let $\mathbb{V}_{h}=V_{h}^{\Omega} \times V_{h}^{\Lambda} \subset \mathbb{V}$ be continuous $k_{1}, k_{2}$-order Lagrangian finite element spaces defined on $\mathcal{T}_{\Omega}^{h}, \mathcal{T}_{\Lambda}^{h}$ respectively. The numerical approximation of the variational formulation (12) consists of finding $\mathcal{U}_{h} \in \mathbb{V}_{h}$ solution of

$$
\mathcal{A}\left(\mathcal{U}_{h}, \mathcal{V}_{h}\right)=\mathcal{F}\left(\mathcal{V}_{h}\right) \quad \forall \mathcal{V}_{h} \in \mathbb{V}_{h}
$$

We notice that in problem (59) it is implicitly assumed that numerical integration is performed exactly. In practice, the average operator $\overline{(\cdot)}$ is approximated by means of numerical quadrature. The effect of the latter approximation shall be analyzed in a future development of this work. Owing to this assumption, the discretization method is consistent and conformal with (12).

For the numerical discretization, we first address the discrete counterparts of (BNB1)-(BNB2), namely,

$$
\begin{gathered}
\exists \alpha_{h}>0: \inf _{\mathcal{W}_{h} \in \mathbb{V}_{h}} \sup _{\mathcal{V}_{h} \in \mathbb{V}_{h}} \frac{\mathcal{A}\left(\mathcal{W}_{h}, \mathcal{V}_{h}\right)}{\left\|\mathcal{W}_{h}\right\|\left|\left\|\mathcal{V}_{h}\right\|\right|} \geq \alpha, \\
\forall \mathcal{V}_{h} \in \mathbb{V}_{h}:\left(\mathcal{A}\left(\mathcal{W}_{h}, \mathcal{V}_{h}\right)=0 \forall \mathcal{W}_{h} \in \mathbb{V}_{h}\right) \Rightarrow \mathcal{V}_{h}=0,
\end{gathered}
$$

which are not necessarily implied by the corresponding continuous properties. As shown in Proposition 2.21 of [15], if the test and the search space of (59) are the same, namely $\mathbb{W}_{h} \equiv \mathbb{V}_{h}$ then $\left(\mathrm{BNB} 1_{h}\right)$ is equivalent to $\left(\mathrm{BNB} 2_{h}\right)$. As a consequence of that, we can verify either $\left(\mathrm{BNB} 1_{h}\right)$ or $\left(\mathrm{BNB} 2_{h}\right)$. We prove the latter using (21) that holds true because of the consistency and the conformity of the method. Owing to this property, $\mathcal{A}\left(\mathcal{V}_{h}, \mathcal{V}_{h}\right)=0$ implies that

$$
\left\|v_{h}\right\|_{H^{1}(\Omega)}=0,\left|V_{h}\right|_{H^{1}(\Lambda),|\mathcal{D}|}=0,\left\|\bar{v}_{h}-V_{h}\right\|_{L^{2}(\Lambda),|\partial \mathcal{D}|}=0,
$$

which is equivalent to $v_{h}=0$ and $\left|V_{h}\right|_{H^{1}(\Lambda),|\mathcal{D}|}=0$ with $\left\|V_{h}\right\|_{L^{2}(\Lambda),|\partial \mathcal{D}|}^{2}=0$, that is $V_{h}=0$.

We exploit the consistency and conformity of the discretization method combined with $\left(\mathrm{BNB} 1_{h}\right)$ and Lemma 3.7, in order to prove that $\mathcal{U}_{h}$ satisfies a Ceá-type inequality ( [15] [Lemma 2.28]),

$$
\left\|\mathcal{U}-\mathcal{U}_{h}\right\| \leq\left(1+\frac{\|\mid \mathcal{A}\|}{\alpha}\right) \inf _{v_{h} \in V_{h}^{\Omega}, V_{h} \in V_{h}^{\Lambda}}\left(\left\|u-v_{h}\right\|_{H^{1}(\Omega)}+\left\|U-V_{h}\right\|_{H^{1}(\Lambda),|\mathcal{D}|}\right) .
$$


The convergence of the finite element method follows from (60) combined with approximation properties of the finite element spaces. For the latter property, we exploit the additional regularity of the solution in $\Omega$ proved in Theorem 3.11 and the fact that the solution $U$ on $\Lambda$ is in $H^{2}(\Lambda)$. The regularity of $U$ descends from the standard properties of elliptic operators in one space dimension, as the right hand side of equation (11b) belongs to $L^{2}(\Lambda)$. From now on, let $a \lesssim b$ be equivalent to the inequality $a \leq C b$ where $C$ is a generic constant, possibly dependent on $\Omega, \Lambda$ but independent of the parameters of the problem. Concerning the solution $u$ in $\Omega$, let $\pi^{h}$ be the Scott-Zhang interpolation operator from $W^{l, q}(\Omega) \cap H_{0}^{1}(\Omega)$ to $V_{h}^{\Omega}$ with $1 \leq q \leq \infty$ and $0 \leq l \leq k_{1}+1$, with the additional constraint $l \geq 1 / q$ when $q>1$. Then, the following interpolation estimate holds true in the norm of $W^{t, q}(\Omega)$ with $t \leq l$ (see for example [15, Lemma 1.130])

$$
\left\|v-\pi^{h} v\right\|_{W^{t, q}(\Omega)} \lesssim h^{l-t}|v|_{W^{l, q}(\Omega)} .
$$

The estimate above applies to the problem at hand, knowing that $u \in H^{\frac{3}{2}-\eta}(\Omega) \cap H_{0}^{1}(\Omega)$, with $t=1$, $l=\frac{3}{2}-\epsilon, q=2, k_{1}=1$, obtaining

$$
\inf _{v_{h} \in V_{h}^{\Omega}}\left\|u-v_{h}\right\|_{H^{1}(\Omega)} \lesssim h^{\frac{1}{2}-\eta}\|u\|_{H^{\frac{3}{2}-\eta}(\Omega)} .
$$

For the solution $U$ on $\Lambda$ and $k_{2}=1$, the standard finite element approximation estimate ensures that

$$
\inf _{V_{h} \in V_{h}^{\Lambda}}\left\|U-V_{h}\right\|_{H^{1}(\Lambda)} \lesssim h\|U\|_{H^{2}(\Lambda)} .
$$

Therefore, combining (60) and the previous inequalities for piecewise affine approximation, we obtain

$$
\left\|\mathcal{U}-\mathcal{U}_{h}\right\| \lesssim h^{\frac{1}{2}-\eta}\|u\|_{H^{\frac{3}{2}-\eta}(\Omega)}+h\|U\|_{H^{2}(\Lambda)}
$$

We have tested the previous convergence results by means of numerical experiments based on a problem for which the analytical solution is known. Precisely, we consider $\Omega=(-1,1)^{3} \subset \mathbb{R}^{3}$ and $\Sigma$ is the cylinder with constant circular cross section of radius $R=0.25$ and centerline $\Lambda=\{(x, 0,0), x \in(-1,1)\}$. We assume $U=1$, therefore the problem reduces to find only the solution $u$ in $\Omega$. Concerning the other parameters, we choose $f=0$ and $\kappa=0.1$. With appropriate boundary conditions, the exact solution $u_{e}$ of the problem can be obtained by uniform extension along the $x$-coordinate the $2 \mathrm{D}$ solution given in [26], which is

$$
u_{e}^{2 D}(y, z)= \begin{cases}U \frac{\kappa}{1+\kappa}\left(1-R \ln \frac{r}{R}(y, z),\right) & r(y, z)>R, \\ U \frac{\kappa}{1+\kappa}, & r(y, z) \leq R,\end{cases}
$$

where $r(y, z)$ is the Euclidean distance from the origin. In particular in our case $u_{e}(x, y, z)=u_{e}^{2 D}(y, z)$. Starting from a quasi-uniform triangulation of $\Omega$ and applying different uniform subdivisions along the $y$ and $z$ axes (denoted as \# sub.), we calculate the discretization error $e_{h}=u_{e}-u_{h}$ and the rate of convergence with respect to the $H^{1}$ - norm. The numerical results reported in Figure 2 agree with the theoretical estimate.

\subsection{A posteriori model error analysis}

Starting from the definitions of the difference operators $d^{(k)}(\cdot, \cdot)$ and $l^{(k)}(\cdot) k=1,2,3$, defined in Sections 4.1, 4.2 and 4.3, we compute a given model error output functional, through the error representation formula (35). In particular, we localize the error on the elements of the triangulation $\mathcal{T}_{\Omega}^{h}$ of $\Omega$. To perform this task, we interpret (35) as the sum of residuals weighed with the dual solution.

First, we introduce the local error estimator using the abstract setting defined at the beginning of Section 4. Let $X_{h} \subset X$ be a suitable finite element space of dimension $N$. We denote with $u_{h}$ and $z_{h \text {,ref }}$ the discrete solutions of the reduced problem and reference dual problem, respectively. Considering the Lagrangian nodal basis $\left\{\phi_{i}\right\} \subset X_{h}$, we define the vector of residuals, $\boldsymbol{\rho}=\left\{\rho_{i}\right\}_{i=1}^{N}$, with $\rho_{i}=l\left(\phi_{i}\right)-d\left(u_{h}\right)\left(\phi_{i}\right)$. According to (35) the local weights, $\omega_{i}$, correspond to the degrees of freedom of the discrete dual solution, namely $z_{h}=\sum_{i=1}^{N} \omega_{i} \phi_{i}$. Let $\langle\cdot, \cdot\rangle$ be the Euclidean scalar product in $\mathbb{R}^{N}$. The model error output functional is then $j(e)=\langle\boldsymbol{\rho}, \boldsymbol{\omega}\rangle$ and the components of the local error estimator $\boldsymbol{\eta} \in \mathbb{R}^{N}$ are $\eta_{i}=\rho_{i} \omega_{i}$. The vector $\boldsymbol{\eta}$ can be 


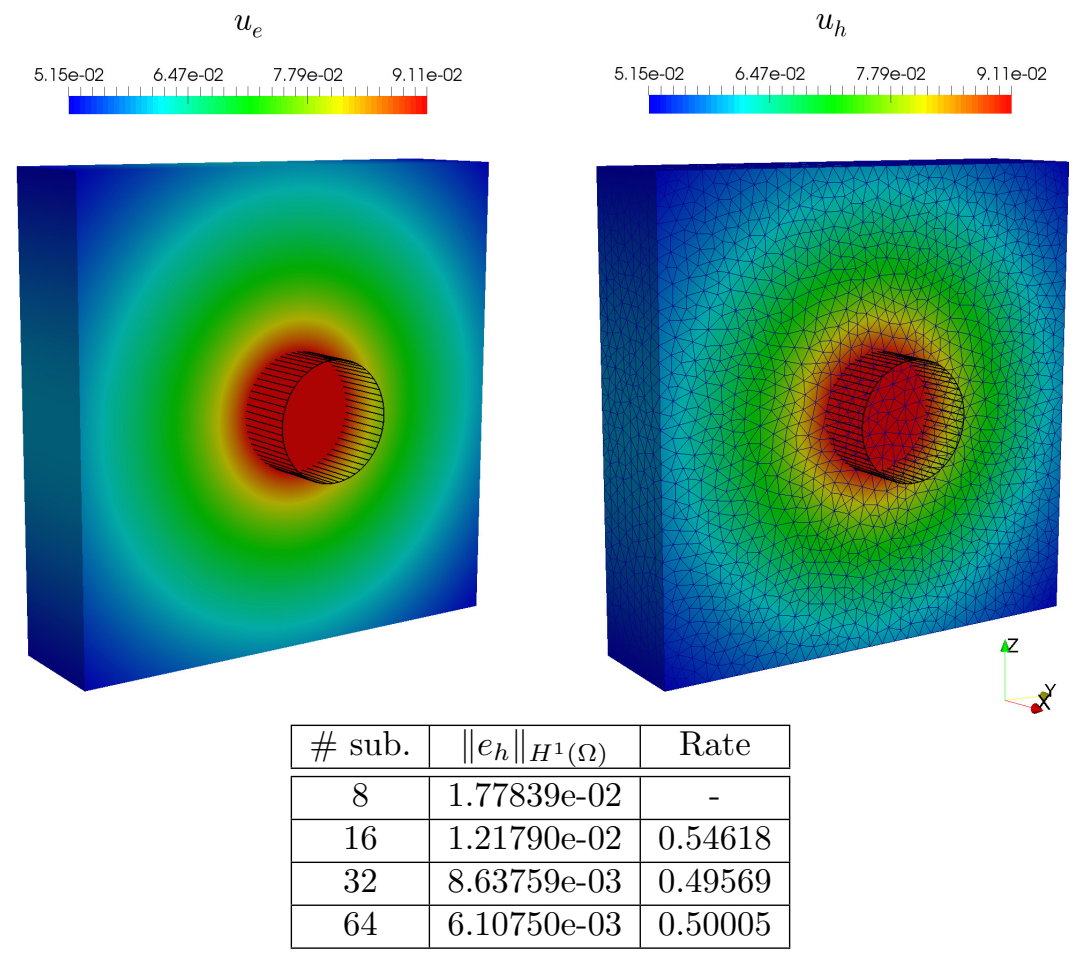

Figure 2. On the top we show a visualization of the exact solution $u_{e}$ and the numerical solution $u_{h}$, superposed to the quasi-uniform mesh used for the convergence test. On the bottom we report the approximation error and the corresponding convergence rate.

represented on $\Omega$ as a finite element function $\eta_{h}=\sum_{i=1}^{N} \eta_{i} \phi_{i}$. Each nodal component of $\boldsymbol{\eta}$ represents the localization of the error on $\mathcal{T}_{\Omega}^{h}$.

Using this general approach we explicitly calculate the estimators $\boldsymbol{\eta}_{1}, \boldsymbol{\eta}_{2}, \boldsymbol{\eta}_{3}$ of the model error of Sections 4.1, 4.2 and 4.3. To this aim, we must compute the residuals $\boldsymbol{\rho}^{(k)}$ and the weights $\boldsymbol{\omega}^{(k)}$ for $k=1,2,3$. For the weights, we recall that $z_{\text {ref }}^{(1)} \in H^{1}(\Sigma), z_{\text {ref }}^{(2)} \in H_{\partial \Omega}^{1}\left(\Omega_{\oplus}\right), z_{\text {ref }}^{(3)} \in H_{0}^{1}(\Omega)$. Then, we introduce $V_{h}^{\Sigma}, V_{h}^{\Omega_{\oplus}}$, $V_{h}^{\Omega}$ the finite element spaces over $\Sigma, \Omega_{\oplus}, \Omega$, respectively. Furthermore, we assume that the following direct sum decomposition holds $V_{h}^{\Omega}=V_{h}^{\Sigma} \oplus V_{h}^{\Omega \oplus}$. In practice, we consider the particular case of a mesh $\mathcal{T}_{\Omega}^{h}$ that nodally conforms with the interface $\Gamma$ and we assign all the nodes on the interface to $V_{h}^{\Omega_{\oplus}}$. According to this notation the discrete reference dual solutions are $z_{h \text {, ref }}^{(1)} \in V_{h}^{\Sigma}, z_{h \text {,ref }}^{(2)} \in V_{h}^{\Omega_{\oplus}}, z_{h, \text { ref }}^{(3)} \in V_{h}^{\Omega}$. However, for computational convenience, we define the all the weight functions on the finite element space $V_{h}^{\Omega} \subset H_{0}^{1}(\Omega)$. Let $N_{h}^{\Omega}=\operatorname{dim}\left(V_{h}^{\Omega}\right)$ be the degrees of freedom of such space and let $\left\{\phi_{i}\right\}_{i=1}^{N_{h}^{\Omega}}$ be the corresponding finite element basis. As a result, all the vectors of weights and residuals belong to $\mathbb{R}_{h}^{N_{h}^{\Omega}}$. Such weights are obtained from the dual solutions by means of the following extensions,

$$
\begin{aligned}
& \omega_{i}^{(1)}= \begin{cases}z_{i}: z_{h, \mathrm{ref}}^{(1)}=\sum_{i=1}^{N_{h}^{\Sigma}} z_{i} \phi_{i} & i=1, \ldots, N_{h}^{\Sigma} \\
0 & i=N_{h}^{\Sigma}+1, \ldots, N_{h}^{\Omega}\end{cases} \\
& \omega_{i}^{(2)}= \begin{cases}0 & i=1, \ldots, N_{h}^{\Sigma} \\
z_{i}: z_{h, \text { ref }}^{(2)}=\sum_{i=1}^{N_{h}^{\Omega} \oplus} z_{i} \phi_{i} & i=N_{h}^{\Sigma}+1, \ldots, N_{h}^{\Omega}\end{cases} \\
& \omega_{i}^{(3)}=z_{i}: \quad z_{h, \text { ref }}^{(3)}=\sum_{i=1}^{N_{h}^{\Omega}} z_{i} \phi_{i} \quad i=1, \ldots, N_{h}^{\Omega} \text {. }
\end{aligned}
$$


We then build the residuals $\boldsymbol{\rho}^{(k)}$ for $k=1,2,3$, depending on the discrete reduced solutions $u_{h}$ and $U_{h}$, computed by means of (59). Using the definitions of (43), (47), (53), for $i=1, \ldots, N_{h}^{\Omega}$ the nodal components of the residuals are,

$$
\begin{aligned}
& \rho_{i}^{(1)}=l^{(1)}\left(\phi_{i}\right)-d^{(1)}\left(U_{h}, \phi_{i}\right)=\left((\mathcal{I}-\overline{\overline{(\cdot)}}) g, \phi_{i}\right)_{\Sigma}+\left(\kappa(\mathcal{I}-\overline{(\cdot)}) u_{h}, \phi_{i}\right)_{\Gamma}, \\
& \rho_{i}^{(2)}=l^{(2)}\left(\phi_{i}\right)-d^{(2)}\left(u_{h}, \phi_{i}\right)=\left(\nabla u_{h}, \nabla \phi_{i}\right)_{\Sigma}-\left(\mathcal{E}_{\Sigma} f, \phi_{i}\right)_{\Sigma}, \\
& \rho_{i}^{(3)}=l^{(3)}\left(\phi_{i}\right)-d^{(3)}\left(u_{h}, \phi_{i}\right)=-\left(\kappa(\mathcal{I}-\overline{(\cdot)}) u_{h}, \phi_{i}\right)_{\Gamma},
\end{aligned}
$$

Finally, we combine the weights and the residuals in order to compute the local estimators,

$$
\begin{aligned}
& \eta_{i}^{(1)}=\omega_{i}^{(1)} \rho_{i}^{(1)}=\omega_{i}^{(1)}\left[\left((\mathcal{I}-\overline{\overline{(\cdot)}}) g, \phi_{i}\right)_{\Sigma}+\left(\kappa(\mathcal{I}-\overline{(\cdot)}) u_{h}, \phi_{i}\right)_{\Gamma}\right], \\
& \eta_{i}^{(2)}=\omega_{i}^{(2)} \rho_{i}^{(2)}=\omega_{i}^{(2)}\left[\left(\nabla u_{h}, \nabla \phi_{i}\right)_{\Sigma}-\left(\mathcal{E}_{\Sigma} f, \phi_{i}\right)_{\Sigma}\right] \\
& \eta_{i}^{(3)}=\omega_{i}^{(3)} \rho_{i}^{(3)}=\omega_{i}^{(3)}\left[-\left(\kappa(\mathcal{I}-\overline{(\cdot)}) u_{h}, \phi_{i}\right)_{\Gamma}\right] .
\end{aligned}
$$

Remark 5.1. We observe that when the mesh nodally conforms with $\Gamma$, the advantage in terms of computational cost of the reduced versus the full (reference) problem almost vanishes. However, we remark that this is a particular case specifically designed to simplify the computations presented below and practically feasible only when the radius of $\Sigma, R$, is comparable with the diameter of $\Omega$.

For the computation of the local model error estimator, we consider the 3D domain $\Omega=(-1,1)^{2} \times$ $(-0.51,0.51)$ and the $1 \mathrm{D}$ segment $\Lambda$ from $(-0.51,0,0)$ to $(0.51,0,0)$. We define on $\Omega$ a a quasi-uniform regular mesh, with characteristic length $h=1 / 32$, for a total of $354 ' 753$ tetrahedra. The $1 \mathrm{D}$ domain is discretized with 1281 points. The parameters of the problem are chosen for simplicity as $R=0.25, k=1$, $f=1$ and $g=1$.

The discrete solutions $u_{h}$ and $U_{h}$ are computed using piecewise linear finite elements. We calculate the residuals $\rho^{(k)}$ by means of $(62)$. We then address the discretization of the reference dual problems (44), (50), (55). To obtain computable solutions, the model error output functionals must not depend on the error itself. We use instead the following definitions,

$$
j^{(1)}(v)=\int_{\Sigma} v d \Sigma, \quad j^{(2)}(v)=\int_{\Omega}\left(\mathcal{I}_{\Omega_{\oplus}}+\mathcal{E}_{\Sigma}\right) v d \Omega, \quad j^{(3)}(v)=\int_{\Omega} v d \Omega .
$$

These output functionals provide the mean value of the error components over $\Sigma$ and $\Omega$, respectively. Given the mesh $\mathcal{T}_{\Omega}^{h}$ that is nodally conforming with the surface $\Gamma$, we define the spaces $V_{h}^{\Sigma}, V_{h}^{\Omega_{\oplus}}, V_{h}^{\Omega}$ using piecewise linear elements, we solve the problems (44), (50), (55) and we compute the weights $w^{(k)}$ using (61). According to (63) the nodal values of the local estimators are the combination of residual and weights. We represent them as piecewise linear finite element functions on $\Omega$.

In what follows, all the results refer to 3D functions in $\Omega$, but the meaningful data are strictly inside the domain, because of the homogeneous Dirichlet condition on his boundary. Therefore, in order to successfully represent the information of the plots, we show only a $2 \mathrm{D}$ slice embedded in the $3 \mathrm{D}$ domain. The bounding box of $\Omega$ is also shown in the plots.

For the discussion of Figure 3 we start from the residual $\rho^{(1)}$, in particular $\left((\mathcal{I}-\overline{\overline{(\cdot)}}) g, \phi_{i}\right)_{\Sigma}$. This term measures the error due to the average on the cross section $\mathcal{D}$ of the forcing term. Since in the simulation of Figure 3, we have used $g=1$ this residual should vanish. A different test using $g=1+3 y$ is addressed in Figure 4 (left column). The comparison among the two cases conforms that, $g$ is variable on $\mathcal{D}$, the residual reproduces the variation of $g$.

Similar considerations apply to the residual $\boldsymbol{\rho}^{(2)}$. It represents the model error due to extending the 3D problem into $\Sigma$. However, even though the magnitude of this residual is the largest among all $\boldsymbol{\rho}^{(k)}, k=1,2,3$, we notice that it is combined with $\boldsymbol{\omega}^{(2)}$, which according to $(61 \mathrm{~b})$ vanishes in the interior of $\Sigma$. As a result, the estimator $\boldsymbol{\eta}^{(2)}$ turns out to be rather small and localized in the neighborhood of the interface.

We also discuss terms on the interface $\left(\kappa(\mathcal{I}-\overline{(\cdot)}) u, \phi_{i}\right)_{\Gamma}$ that appear in $\boldsymbol{\rho}^{(1)}$ and $\boldsymbol{\rho}^{(3)}$. These residuals depend on the function $f$. Precisely, if $f$ is constant, then also the solution $u$ does not vary along $\partial \mathcal{D}$ and 

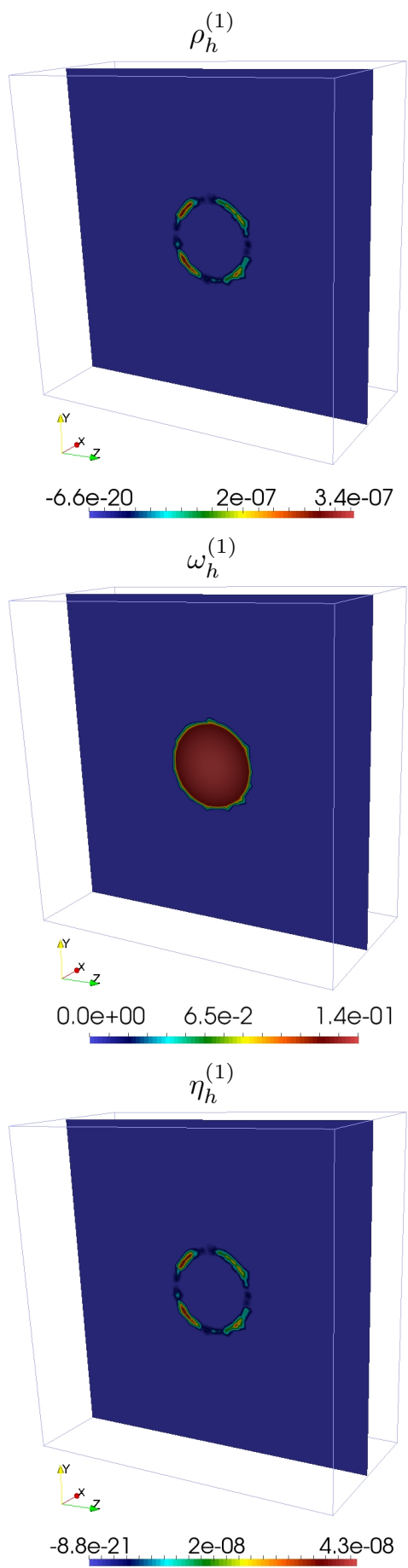
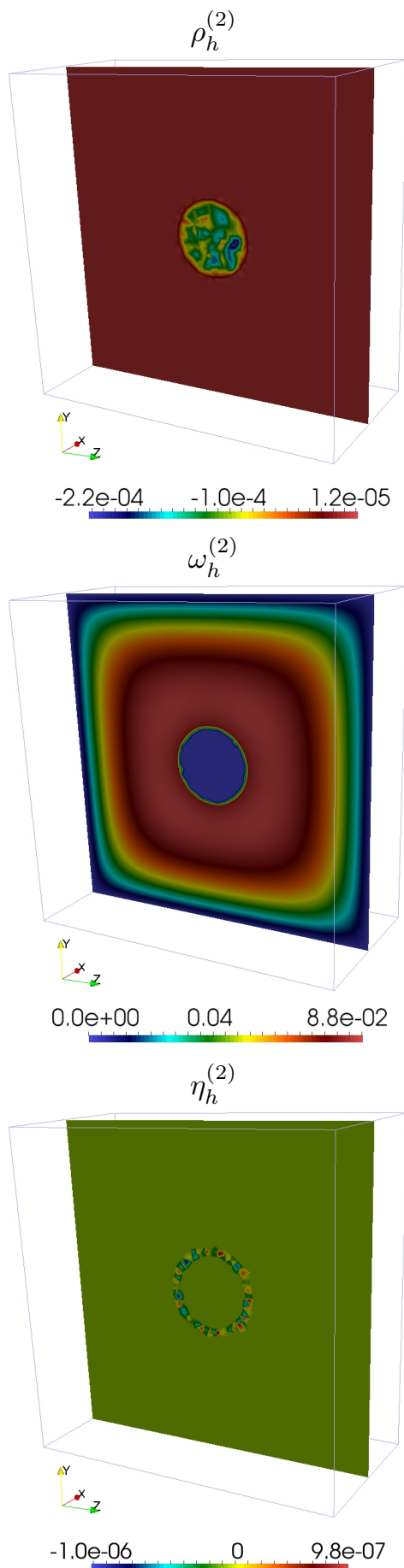
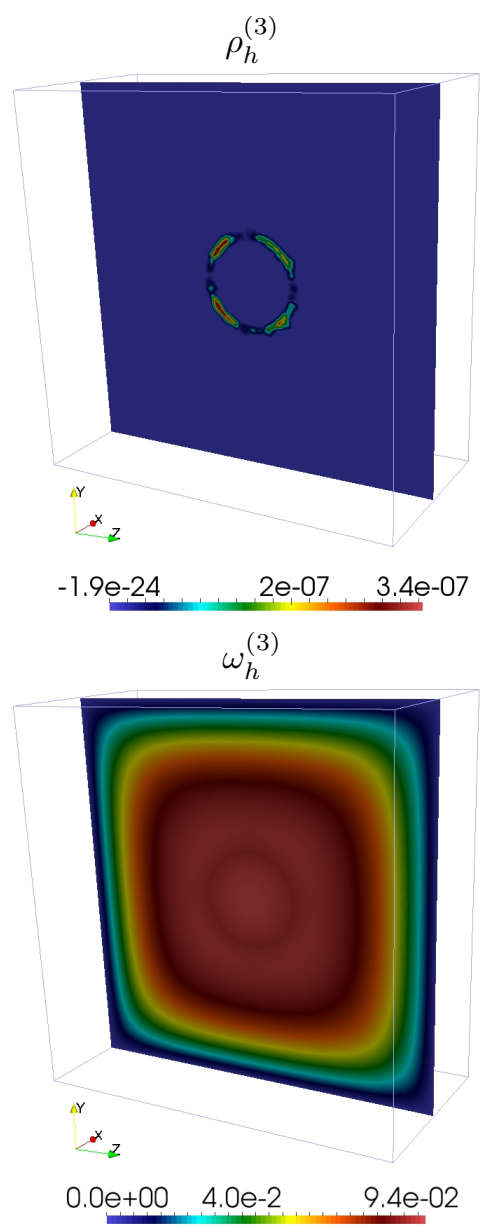

$\eta_{h}^{(3)}$

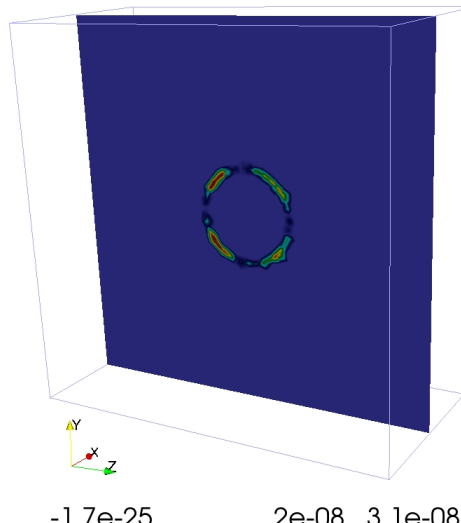

$-1.7 e-25 \quad 2 e-08 \quad 3.1 e-08$

FIGURE 3. Analysis of the residuals $\rho_{h}^{(k)}$, the weights $\omega_{h}^{(k)}$ and the local estimators $\eta_{h}^{(k)}$, represented as piecewise linear functions on the mesh $\mathcal{T}_{\Omega}^{h}$.

$u(s)=\bar{u}(s)$. Therefore, the residuals $\boldsymbol{\rho}^{(1)}$ and $\boldsymbol{\rho}^{(3)}$ become relevant especially when $f$ is not constant around the inclusion. The comparison of these residuals in the case $f=1$ and $f=1+y$, shown in Figure 4 (right column), confirms the expected behavior. 

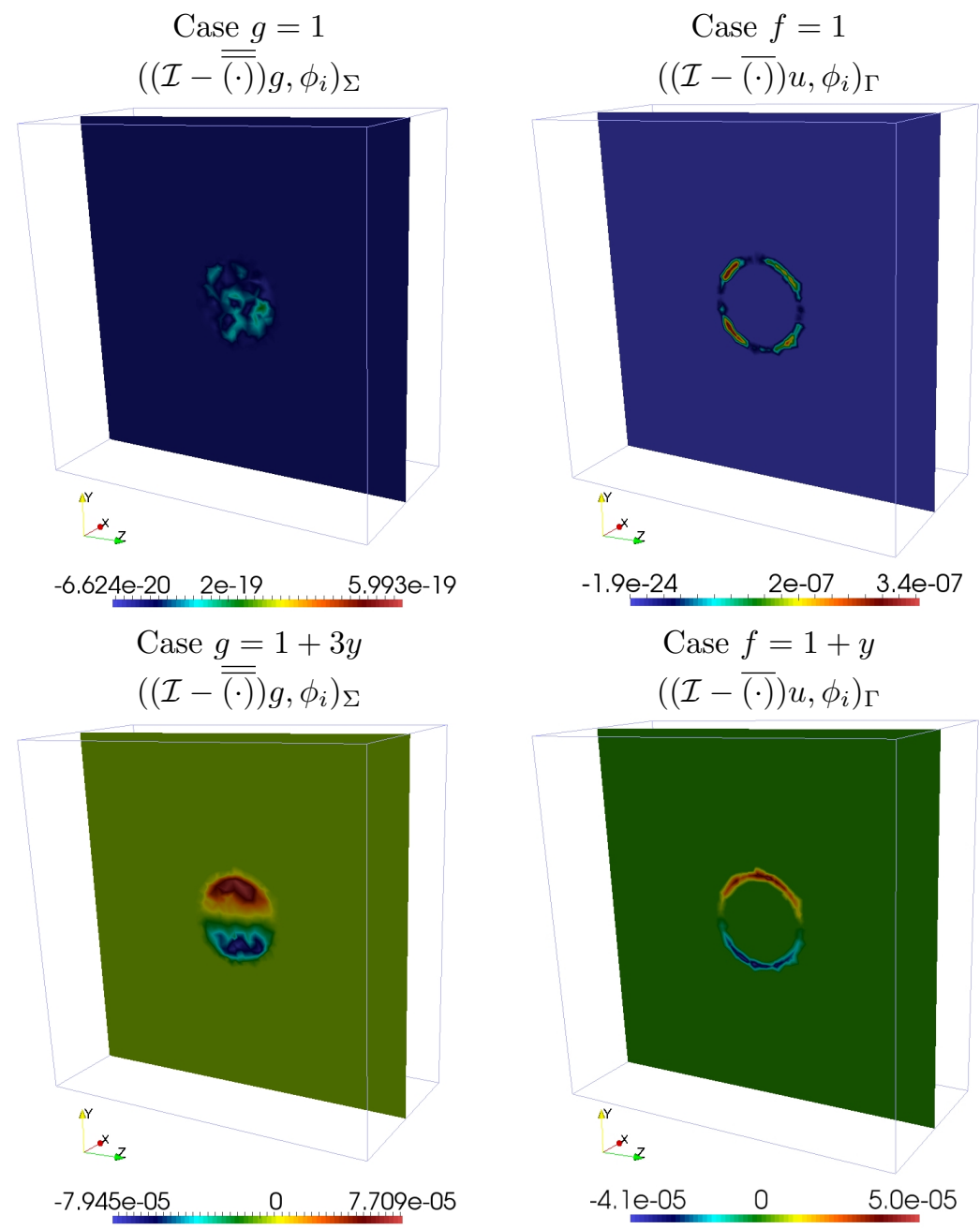

FiguRE 4. On the left column we show the comparison of $\left(g, \phi_{i}\right)_{\Sigma}$ and $\left.(\overline{\overline{(\cdot)}}) g, \phi_{i}\right)_{\Sigma}$ in the case $g=1$ and $g=1+3 y$. On the right, we analyze the comparison of $\left(u, \phi_{i}\right)_{\Gamma}$ and $\left(\bar{u}, \phi_{i}\right)_{\Gamma}$ in the case $f=1$ and $f=1+y$.

Finally, we consider a test case with a thinner inclusion, namely a cylinder with radius $R=0.1$. The domains $\Omega$ and $\Lambda$ as well as the parameters $k, f$ and $g$ are the same as in the previous case shown in Figure 3. The computational mesh has been slightly refined in the neighborhood of the cylinder, to comply with its size. The visual analysis of the error estimator (not shown here) confirms that the spatial distribution of the error is the same as the one of Figure 3. In Table 1 we show a quantitative comparison of the two cases. As expected, the results confirm that the model error decreases with the size of the inclusion.

\section{Conclusions}

This work illustrates the foundations of a multiscale method for solving partial differential equations on bulk domains with embedded network-shaped cylindrical inclusions. The method consists of applying a model reduction technique first, aiming at transforming the original problem into a simpler one. Such problem features equations in a bulk domain coupled with a collection of one-dimensional problems. After showing the well posedness of the reduced problem, we have approximated it by means of finite elements and proved the convergence of the discretization scheme. Finally, we have pursued the analysis of the modeling error, namely the difference between the solutions of the original problem and the simplified one. We have 


\begin{tabular}{|c|c|c|c|c|c|c|}
\hline & \multicolumn{2}{|c|}{$j^{(1)}(e)=\sum_{i} \eta_{i}^{(1)}$} & \multicolumn{2}{|c|}{$j^{(2)}(e)=\sum_{i} \eta_{i}^{(2)}$} & \multicolumn{2}{|c|}{$j^{(3)}(e)=\sum_{i} \eta_{i}^{(3)}$} \\
\hline $\bar{R} R=0.25$ & \multicolumn{2}{|c|}{$2 \mathrm{e}-04$} & \multicolumn{2}{|c|}{$-1 \mathrm{e}-02$} & \multicolumn{2}{|c|}{$-1 \mathrm{e}-05$} \\
\hline$R=0.1$ & \multicolumn{2}{|c|}{$9 \mathrm{e}-07$} & \multicolumn{2}{|c|}{$-3 e-05$} & \multicolumn{2}{|c|}{$-1 \mathrm{e}-06$} \\
\hline & \multicolumn{2}{|c|}{$\overline{\overline{\eta^{(1)}}}$} & \multicolumn{2}{|c|}{$\boldsymbol{\eta}^{(2)}$} & \multicolumn{2}{|c|}{$\boldsymbol{\eta}^{(3)}$} \\
\hline & $\min _{i}$ & $\max _{i}$ & $\min _{i}$ & $\max _{i}$ & $\min _{i}$ & $\max _{i}$ \\
\hline$R=0.25$ & $-8 \mathrm{e}-21$ & $4 \mathrm{ee}-08$ & $-1 \mathrm{e}-05$ & $9 \mathrm{e}-07$ & $-1 \mathrm{e}-25$ & $3 \mathrm{e}-08$ \\
\hline$R=0.1$ & $-1 \mathrm{e}-11$ & $4 \mathrm{e}-10$ & $-8 \mathrm{e}-09$ & $1 \mathrm{e}-07$ & $-3 \mathrm{e}-11$ & $8 \mathrm{e}-10$ \\
\hline
\end{tabular}

TABLE 1. Variation of the error output functionals $j^{(k)}(e)$ and of the error estimators $\boldsymbol{\eta}^{(k)}$ when the radius of the inclusion $\Sigma$ decreases from $R=0.25$ to $R=0.1$.

shown that for infinitesimally narrow inclusions, representing for example a bulk material perfused by narrow channels, the reduced model converges to the original one. Although realistic applications require to address more complicated equations, which have not been fully analyzed yet in this context, we believe that the sound mathematical properties proved here strengthen the significance of this approach to applications. However, there are still many open questions to be addressed. For example, this work should be extended to other types of interface conditions among the bulk and the inclusions, addressing for example Dirichlet type constraints. In the same spirit, we are studying the method for partial differential equations in mixed form, in order to better model flow problems. Important and pressing questions also arise at the level of numerical discretization and solvers. For example, the role of quadrature in the approximation of the averaging operators on the global accuracy of the approach is still unexplored. At the level of numerical solver, efficient solution methods and preconditioners for coupled partial differential equations on embedded domains must be investigated.

\section{REFERENCES}

[1] Serge Alinhac and Patrick Gérard. Pseudo-differential operators and the Nash-Moser theorem, volume 82 of Graduate Studies in Mathematics. American Mathematical Society, Providence, RI, 2007. Translated from the 1991 French original by Stephen S. Wilson.

[2] Mario Arioli and Michele Benzi. A finite element method for quantum graphs. IMA J. Numer. Anal., 38(3):1119-1163, 2018.

[3] Gregory Berkolaiko, Robert Carlson, Stephen A. Fulling, and Peter Kuchment. Quantum graphs and their applications, volume 415 of Contemporary Mathematics. American Mathematical Society, Providence, RI, 2006.

[4] S. Bertoluzza, A. Decoene, L. Lacouture, and S. Martin. Local error estimates of the finite element method for an elliptic problem with a Dirac source term. Numerical Methods for Partial Differential Equations, 34(1):97-120, 2018.

[5] T.R. Blake and J.F. Gross. Analysis of coupled intra- and extraluminal flows for single and multiple capillaries. Mathematical Biosciences, 59(2):173-206, 1982.

[6] Wietse Boon, Jan Nordbotten, and Jon Vatne. Functional analysis and exterior calculus on mixed-dimensional geometries. Technical report, arXiv, Cornell University Library, 2018. arXiv:1710.00556v3.

[7] Malte Braack and Alexandre Ern. A posteriori control of modeling errors and discretization errors. Multiscale Model. Simul., 1(2):221-238, 2003.

[8] Susanne C. Brenner. Poincaré-Friedrichs inequalities for piecewise H1 functions. SIAM Journal on Numerical Analysis, 41(1):306-324, 2003.

[9] L. Cattaneo and P. Zunino. A computational model of drug delivery through microcirculation to compare different tumor treatments. International Journal for Numerical Methods in Biomedical Engineering, 30(11):1347-1371, 2014.

[10] L. Cattaneo and P. Zunino. Computational models for fluid exchange between microcirculation and tissue interstitium. Networks and Heterogeneous Media, 9(1):135-159, 2014.

[11] D. Cerroni, F. Laurino, and P. Zunino. Mathematical analysis, finite element approximation and numerical solvers for the interaction of 3d reservoirs with 1d wells. GEM - International Journal on Geomathematics, 10(1), 2019.

[12] C. D'Angelo. Multi scale modelling of metabolism and transport phenomena in living tissues, PhD Thesis. EPFL, Lausanne, 2007.

[13] C. D'Angelo. Finite element approximation of elliptic problems with Dirac measure terms in weighted spaces: applications to one- and three-dimensional coupled problems. SIAM Journal on Numerical Analysis, 50(1):194-215, 2012.

[14] C. D'Angelo and A. Quarteroni. On the coupling of $1 \mathrm{~d}$ and 3d diffusion-reaction equations. Application to tissue perfusion problems. Mathematical Models and Methods in Applied Sciences, 18(8):1481-1504, 2008. 
[15] Alexandre Ern and Jean-Luc Guermond. Theory and practice of finite elements, volume 159 of Applied Mathematical Sciences. Springer-Verlag, New York, 2004.

[16] Julián Fernández Bonder and Julio D. Rossi. Asymptotic behavior of the best Sobolev trace constant in expanding and contracting domains. Commun. Pure Appl. Anal., 1(3):359-378, 2002.

[17] G.J. Fleischman, T.W. Secomb, and J.F. Gross. The interaction of extravascular pressure fields and fluid exchange in capillary networks. Mathematical Biosciences, 82(2):141-151, 1986.

[18] G.J. Flieschman, T.W. Secomb, and J.F. Gross. Effect of extravascular pressure gradients on capillary fluid exchange. Mathematical Biosciences, 81(2):145-164, 1986.

[19] I. Gansca, W. F. Bronsvoort, G. Coman, and L. Tambulea. Self-intersection avoidance and integral properties of generalized cylinders. Comput. Aided Geom. Design, 19(9):695-707, 2002.

[20] David Gilbarg and Neil S. Trudinger. Elliptic partial differential equations of second order. Classics in Mathematics. Springer-Verlag, Berlin, 2001. Reprint of the 1998 edition.

[21] Ingeborg Gjerde, Kundan Kumar, Jan M. Nordbotten, and Barbara Wohlmuth. Splitting method for elliptic equations with line sources. ESAIM: Mathematical Modelling and Numerical Analysis, pages 1-26, 2019. Accepted article in print.

[22] W. Gong, G. Wang, and N. Yan. Approximations of elliptic optimal control problems with controls acting on a lower dimensional manifold. SIAM Journal on Control and Optimization, 52(3):2008-2035, 2014.

[23] T. Koch, K. Heck, N. Schrder, H. Class, and R. Helmig. A new simulation framework for soilroot interaction, evaporation, root growth, and solute transport. Vadose Zone Journal, 17(1), 2018.

[24] T. Köppl, E. Vidotto, and B. Wohlmuth. A local error estimate for the Poisson equation with a line source term. In Numerical Mathematics and Advanced Applications ENUMATH 2015, pages 421-429. Springer, 2016.

[25] T. Köppl and B. Wohlmuth. Optimal a priori error estimates for an elliptic problem with Dirac right-hand side. SIAM Journal on Numerical Analysis, 52(4):1753-1769, 2014.

[26] Tobias Köppl, Ettore Vidotto, Barbara Wohlmuth, and Paolo Zunino. Mathematical modeling, analysis and numerical approximation of second-order elliptic problems with inclusions. Mathematical Models and Methods in Applied Sciences, 28(05):953-978, 2018.

[27] M. Kuchta, M. Nordaas, J.C.G. Verschaeve, M. Mortensen, and K.-A. Mardal. Preconditioners for saddle point systems with trace constraints coupling 2d and 1d domains. SIAM Journal on Scientific Computing, 38(6):B962-B987, 2016.

[28] Miroslav Kuchta, Kent-Andre Mardal, and Mikael Mortensen. Preconditioning trace coupled $3 d-1 d$ systems using fractional Laplacian. Numer. Methods Partial Differential Equations, 35(1):375-393, 2019.

[29] J. R. Kuttler and V. G. Sigillito. An inequality of a Stekloff eigenvalue by the method of defect. Proc. Amer. Math. Soc., 20:357-360, 1969.

[30] M. Lesinigo, C. D'Angelo, and A. Quarteroni. A multiscale Darcy-Brinkman model for fluid flow in fractured porous media. Numerische Mathematik, 117(4):717-752, 2011.

[31] M. Nabil, P. Decuzzi, and P. Zunino. Modelling mass and heat transfer in nano-based cancer hyperthermia. Royal Society Open Science, 2(10), 2015.

[32] M. Nabil and P. Zunino. A computational study of cancer hyperthermia based on vascular magnetic nanoconstructs. Royal Society Open Science, 3(9), 2016.

[33] D. Notaro, L. Cattaneo, L. Formaggia, A. Scotti, and P. Zunino. A Mixed Finite Element Method for Modeling the Fluid Exchange Between Microcirculation and Tissue Interstitium, pages 3-25. Springer International Publishing, 2016.

[34] L. E. Payne and H. F. Weinberger. An optimal Poincaré inequality for convex domains. Arch. Rational Mech. Anal., 5:286-292 (1960), 1960.

[35] Donald W. Peaceman. Interpretation of well-block pressures in numerical reservoir simulation with nonsquare grid blocks and anisotropic permeability. Society of Petroleum Engineers journal, 23(3):531-543, 1983.

[36] D.W. Peaceman. Interpretation of well-block pressures in numerical reservoir simulation. Soc Pet Eng AIME J, 18(3):183194, 1978.

[37] L. Possenti, G. Casagrande, S. Di Gregorio, P. Zunino, and M.L. Costantino. Numerical simulations of the microvascular fluid balance with a non-linear model of the lymphatic system. Microvascular Research, 122:101-110, 2019.

[38] L. Possenti, S. di Gregorio, F.M. Gerosa, G. Raimondi, G. Casagrande, M.L. Costantino, and P. Zunino. A computational model for microcirculation including Fahraeus-Lindqvist effect, plasma skimming and fluid exchange with the tissue interstitium. International Journal for Numerical Methods in Biomedical Engineering, 35(3), 2019.

[39] A. Quarteroni, A. Veneziani, and C. Vergara. Geometric multiscale modeling of the cardiovascular system, between theory and practice. Computer Methods in Applied Mechanics and Engineering, 302:193-252, 2016.

[40] G. Raimondi. Computational models for root water uptake. Master's thesis, Politecnico di Milano, 2017.

[41] S. A. Sauter and R. Warnke. Extension operators and approximation on domains containing small geometric details. East-West J. Numer. Math., 7(1):61-77, 1999.

[42] T. Secomb, R. Hsu, E. Park, and M. Dewhirst. Green's function methods for analysis of oxygen delivery to tissue by microvascular networks. Annals of Biomedical Engineering, 32(11):1519-1529, 2004.

[43] Michael Solomyak. On approximation of functions from Sobolev spaces on metric graphs. J. Approx. Theory, 121(2):199$219,2003$.

[44] A.-K. Tornberg and B. Engquist. Numerical approximations of singular source terms in differential equations. Journal of Computational Physics, 200(2):462-488, 2004. 